\title{
Notch ligand Dll4 impairs cell recruitment into aortic clusters and limits hematopoietic stem cells
}

Cristina Porcheri ${ }^{1}$, Ohad Golan ${ }^{2}$, Fernando J. Calero-Nieto ${ }^{3}$, Roshana Thambyrajah ${ }^{1}$, Cristina Ruiz-Herguido ${ }^{1}$, Xiaonan Wang ${ }^{3}$, Francesca Catto ${ }^{1}$, Yolanda Guillen ${ }^{1}$, Roshani Sinha ${ }^{1}$, Jessica González $^{1}$, Sarah J. Kinston ${ }^{3}$, Samanta A. Mariani ${ }^{4}$, Antonio Maglitto ${ }^{4}$, Chris Vink ${ }^{4}$, Elaine Dzierzak $^{4}$, Pierre Charbord ${ }^{5}$, Bertie Göttgens ${ }^{3}$, Lluis Espinosa ${ }^{1}$, David Sprinzak ${ }^{2}$, Anna Bigas $^{1 *}$

${ }^{1}$ Program in Cancer Research. Institut Hospital del Mar d'Investigacions Mèdiques, CIBERONC, Barcelona, Spain

${ }^{2}$ Department of Biochemistry and Molecular Biology, Wise Faculty of Life Science, Tel Aviv University, Tel Aviv, 69978, Israel

${ }^{3}$ Wellcome and MRC Cambridge Stem Cell Institute, CIMR, Hills Road, Cambridge CB2 OXY, UK

${ }^{4}$ Centre for Inflammation Research, University of Edinburgh, UK

${ }^{5}$ Department of Developmental biology. Institute of Biology of Paris Seine (IBPS). Sorbonne University, France.

Running title: DII4 shapes the niche of HSC development

Keywords: AGM, HSC, hemogenic endothelium, Notch, DIl4

*Corresponding author:

Anna Bigas, Cancer Research Program. CIBERONC.

Institut Mar d'Investigacions Mèdiques,

Doctor Aiguader 88, 08003, Spain.

Tel: +34 933160589; email, abigas@imim.es.

Authors can confirm that all relevant data are included in the paper and/or its supplementary information files. The datasets generated and analysed during the current study are available from the corresponding author on reasonable request. The RNAseq and scRNAseq datasets generated and analysed during the current study (Figure 5 and Supplementary Figure 5) are available in the GEO repository, with the accession codes (submitted). 


\begin{abstract}
Hematopoietic stem cells (HSCs) develop from the hemogenic endothelium in cluster structures that protrude into the embryonic aortic lumen. Although much is known about the molecular characteristics of the developing hematopoietic cells, we lack a complete understanding of their origin and the three-dimensional organization of the niche. Here we use advanced live imaging techniques of organotypic slice cultures, clonal analysis, and mathematical modelling to show the two-step process of intra-aortic hematopoietic cluster (IACH) formation. First, a hemogenic progenitor buds up from the endothelium and undergoes division forming the monoclonal core of the IAHC. Next, surrounding hemogenic cells are recruited into the IAHC, increasing their size and heterogeneity. We identified the Notch ligand DII4 as a negative regulator of the recruitment phase of IAHC. Blocking of DII4 promotes the entrance of new hemogenic Gfi1+ cells into the IAHC and increases the number of cells that acquire HSC activity. Mathematical modelling based on our data provides estimation of the cluster lifetime and the average recruitment time of hemogenic cells to the cluster under physiologic and DII4-inhibited conditions.
\end{abstract}




\section{INTRODUCTION}

Hematopoietic stem cells (HSCs) are generated during embryonic life in a process known as endothelial to hematopoietic transition (EHT). EHT takes place in several vessels of the vertebrate embryo and involves the acquisition of hematopoietic features by a specific population of endothelial cells ${ }^{1-4}$. However, most of the cells that undergo EHT become differentiated hematopoietic cells or progenitors, but do not become HSCs. A number of cells localized in the dorsal aorta in the AGM region (where AGM refers to the Aorta surrounded by Gonads and Mesonephros tissues) will generate HSCs ${ }^{5,6}$ and will be the founders of the adult hematopoietic system. Within the AGM region, and in the ventral side of the aorta, a subset of endothelial cells that is characterized by the expression of hemogenic factors such as Gfi1, Runx1 and/or Gata2 are collectively known as hemogenic endothelium. In most vertebrate embryos, functional HSCs develop within hematopoietic clusters that derive from the hemogenic endothelium and then emerge into the lumen of the aorta ${ }^{4}$. Intra-aortic hematopoietic clusters (IAHC) contain and are the niche of nascent HSCs in most vertebrates, however the mechanisms of IAHC formation and HSC development inside these structures remain elusive. It was previously postulated that bulging of endothelial cells expressing CD41, Kit, Gata2 and/or Gfi1 amongst others, is one of the first events in the formation of hematopoietic clusters ${ }^{7-10}$. Recent reports studying clonality of cells in the IAHC have determined that they are polyclonal in origin suggesting that at least two independently generated cells aggregate to form a cluster ${ }^{5,11}$. In addition, cells in the IAHC are cycling and highly proliferative ${ }^{11,12}=$

Beyond these descriptive analyses, the events, mechanisms, and dynamics that determine the architecture of the clusters have never been addressed. Previous published work on signals required for HSC generation converge on the Notch pathway (reviewed in ${ }^{13}$ ). Notch receptors can be activated by different ligands either of the Jagged or Delta family of proteins. In the embryonic aortic endothelium, both types of ligands are expressed in a variety of cells and their interplay seems to be crucial to initiate the arterial and hematopoietic programs) ${ }^{14-17}$.

Here we show by confocal live imaging, clonal genetic studies, single cell RNA-seq and mathematical modeling that IAHCs are originated as clonal entities by cell division, but soon 
after the first division event, new neighboring cells are recruited into the cluster. We found that the number of cells inside the clusters is tightly controlled by the Notch ligand DII4, which restrains the recruitment of new cells in the newly-formed clusters. Blockage of DII4 results in larger polyclonal clusters and affects the number of functional HSCs generated.

\section{RESULTS}

\section{Formation of IAHC initiates with cell division}

To better understand the formation of IAHC, we imaged the first stages of cluster emergence using a reporter for CD41 (Integrin all $\beta$ ), which is one of the earliest genes expressed in the hematopoietic clusters together with kit (Fig1b) ${ }^{9}$. We crossed membrane tethered CD41:YFP reporter mice ${ }^{18}$ with the $\mathrm{H} 2 \mathrm{~B}-\mathrm{GFP}$ transgenic line ${ }^{19}$ and performed time-lapse imaging on organotypic slice culture of E10.5 double transgenic embryos. We consistently identified single CD41:YFP+ cells arising from the endothelial layer and protruding onto the aortic lumen (Fig1a, Fig1c, Movie 1). In agreement with previous observations ${ }^{4}$, we only detected individual cells, and not groups of CD41:YFP+ cells bulging towards the lumen of the aorta. Time-lapse analysis revealed that individual bulging CD41:YFP+ cells subsequently undergo a cellular division (Fig1a,Fig1d, Movie2) and both CD41:YFP+ daughter cells remained in intimate contact, likely forming the first unit of the IAHC. Within the IAHC, hematopoietic cells are actively cycling (Fig1e, Fig1f, Suppl Fig1), and incorporate BrdU upon continuous administration, until all cells in the clusters are positive (saturation time: 7-8h, Fig1f). Considering that the percentage of BrdU-incorporating cells does not change between 1 and 4 hours of initial BrdU exposure, we assume that the S-phase length (Ts) lasts 4-5 hours. This allows us to estimate the cell cycle length (Tc) duration in 11-13 hours, as a result of the sum between the saturation time $(7-8 h)$ and the estimated Ts $(4-5 h)^{20}$. To confirm that all cells in the IAHC are actively cycling, we analyzed the rate of Ki67-expression to find a high number of Ki67 positive cells in the clusters $(87,4 \% \pm 5,2)$ compared to the $(59,3 \% \pm 2,92)$ of the neighboring endothelial cells (Suppl Fig1a, 1b, 1c and 1d). Time-lapse imaging of mitotic 
CD41:YFP+ cells and staining for the P-Histone3 mitotic marker confirms the existence of cell division inside IAHCs (Suppl Fig 2, Movie3).

\section{IAHCs start monoclonal but become polyclonal after cell division.}

To test whether hematopoietic clusters were formed by a series of sequential divisions from a single hemogenic precursor, we took advantage of the Confetti reporter mouse crossed with the VE-cadherin-Cre-ERT (VeCadCre ${ }^{\mathrm{ER}}$ ) line. In this model, single pulse administration of Tamoxifen before the time of cluster emergence (E8), allows Cre translocation and recombination of the transgene in cells expressing VE-Cadherin, including the hemogenic endothelial cells that will give rise to clusters (Fig 2a). In our experimental conditions, the overall recombination efficiency of the confetti cassette in the endothelial cells was of $22.92 \pm$ $5.4 \%$ (Fig 2b and Fig2c) and allowed the tracking of the sparse single recombined cells. Clusters of monoclonal origin would display one single color (from the originating cell), while in case of a polyclonal origin the cluster will contain cells of various colors or a mix of colored and uncolored cells (Fig 2d, Fig 2e). After labeling cluster cells with the Kit marker, we consistently found that two-cell and three-cell clusters were all single-colored (monoclonal), while clusters with 4 or more cells mostly contained heterogeneous labeling patterns suggestive of a polyclonal origin (Fig $2 \mathrm{f}$ and Fig $2 \mathrm{~g}$ ). These results indicated that IAHC formation starts with the generation of a monoclonal core and continues growth with cells from other sources.

\section{The Notch ligand DII4 is preferentially expressed in small IAHC}

Because the Notch pathway is crucial for HSC development ${ }^{21,22}$, we performed a detailed study of the expression levels and distribution of Notch elements in the AGM. We found a highly heterogeneous expression of Dll4 ligand in most IAHC and endothelial cells (Fig 3). Confocal microscopy analysis demonstrated that small clusters (2-5 cells) were exclusively formed by Dll4 positive cells, while bigger clusters (>5 cells) contained an increasing proportion of Dll4 negative cells (Fig 3B). In clusters with more than 11 Kit+ cells only $47 \% \pm 10$ express the Notch ligand DII4 (Fig 3A and 3B). In these clusters, 42\% showed Dll4+ cells located in the apical portion toward the lumen (Figure 3A), however we could not establish a 
common pattern since the rest of the analyzed IAHC had DII4+ cells randomly distributed between basal (endothelial) and apical portion (lumen). In contrast, the Notch ligand Jag1, which is required for downregulation of the endothelial program ${ }^{23}$ and Notch-dependent activation of hematopoietic genes in the nascent HSCs ${ }^{22}$ was found preferentially expressed in the endothelial layer of the AGM (Suppl Fig 3). These results suggest that DII4 is not only required to promote the arterial fate as previously demonstrated, but also plays a role in IAHC formation.

\section{Specific blockage of DII4 results in increased IAHC size.}

To investigate the functional contribution of DII4 in the IAHCs, we used an $\alpha$ DII4 antibody specifically developed to interfere with the DII4-dependent activation of Notch ${ }^{24}$. We injected $\alpha$ Dll4 or IgG control into the beating heart of the E10-E10.5 embryos (32-37 sp) to minimize the potential side effects of a systemic administration. Following this procedure, we found that the antibody was distributed according to physiologic blood flow and specifically recognized the Dll4-ligand exposed to the aortic lumen (Suppl Fig 4). We then maintained the injected embryos in organotypic culture conditions for 5 hours and processed them for subsequent analyses. Whole mount immunofluorescence of CD31 and Kit staining revealed that the structure of the aorta was preserved after the incubation with aDII4 comparable to IgG control injected embryos (Fig 4a). Kit+ IAHC were similarly distributed along the aorta of control and $\alpha$ DII4 treated embryos while larger IAHCs were found in the latter (Fig 4a, Fig 4b). We then analyzed the size of each cluster per embryo based on the number of Kit+ cells forming them (Fig 4b, Fig 4c, Fig 4d). In control-treated embryos, the majority of IAHC were small (in the range of 2 to 5 cells: $80 \pm 3 \%$ ) and no cluster above 18 cells was detected. In contrast, in the $\alpha$ DII4-treated embryos, the percentage of small IAHC decreased (53. $\pm 8 \%$ ) while bigger clusters were more frequently found, with very large IAHCs (>16 cell) representing $5 \pm 3 \%$ of total clusters (Fig 4 c, $4 d$ ). Interestingly, the total number of IAHCs (Fig $4 \mathrm{e}$ ) or the single $\mathrm{kit}^{+}$cells bulging from the endothelium (Fig 4f) did not change upon $\alpha \mathrm{DIl} 4$ treatment, suggesting that DII4 function was confined to the already initiated IAHCs.

\section{Multiple signaling pathways including Notch are altered in Dll4-blocked Kit+CD45- cells}


To investigate the mechanism behind the increased cellularity of IAHC after Dll4 blockage, we purified IAHC subpopulations based on kit expression. In particular, we collected 100-300 cells from the Kit+CD45- (containing Pre-HSCs), Kit+CD41+CD45- and Kit+CD45+ (containing HSCs) from 4 different IgG and $\alpha$ DII4 treated embryos and performed RNA-seq (Fig 5a). Differentially expressed genes (DEGs) among the three different populations were assessed by pairwise comparisons, resulting in three sets of up- and down-regulated genes. Intersection of the gene sets allowed discriminating DEGs according to the treatment that were exclusive to each cell population. Principal Component Analysis (PCA) using the total number of DEGs indicated that the samples clearly segregated according to phenotype (Fig 5B). In a second comparison we contrasted control vs $\alpha$ DII4 treated cells and performed a PCA using the DEGs that drove a clear-cut discrimination between treated and untreated samples (Fig 5C). Moreover, both PCA plots revealed that the Kit+CD45- pre-HSC-containing population showed the clearest clusterization based on the treatment. A functional enrichment analysis on the DEGs according to the treatment is shown in Fig 5D. Within the biological processes enriched in dowregulated genes after $\alpha$ DII4 treatment, we found the Notch signaling pathway. In addition to Notch, crucial pathways for HSC development such as NFKB, Wnt and TGF $\beta$ pathways were specifically enriched after the treatment, as well as alterations in categories associated with cell remodeling and movement (Fig 5d). Among up-regulated genes, there were genes involved in RNA processing and DNA repair.

To further investigate the effect of Dll4 intervention in individual Kit+ precursors, we performed single cell RNA-seq of CD31+kit+CD45- cells (Fig 5e). We analyzed 239 control cells and 244 aDll4 -treated cells from 4 different embryos each (4 untreated (32-34sp) and 4 treated (32-35sp)). Three subpopulations were clearly separated by tSNE, which correlated with 7 cell clusters generated by Iterative Clustering and Guide-gene Selection (ICGS, ${ }^{25}$ ) (Fig 5g and Suppl Fig 5a and Suppl Table 1). Based on marker gene expression, the largest cell population corresponded to endothelium while hematopoietic marker gene expression was more prominent in the smaller population. Importantly, integrated analysis with scRNA-Seq from FACS-sorted AGM populations ${ }^{26}$ confirmed the endothelial and hematopoietic nature of these populations (Suppl. Fig 5b and Suppl Table 1). While there was no readily identifiable shift in cell populations after blocking DII4, differential gene expression between IgG and 
$\alpha$ Dll4-treated Kit+ cells (Fig 5f) revealed downregulation of several genes associated with the Notch pathway (Fig 5h) and other signaling pathways (Suppl Table 2). Importantly the significance of this observation was confirmed by gene pathway enrichment analysis (Suppl. Fig 5c). Of note, computational analysis of single cell transcriptomes revealed no change in the number of cells in different phases of the cell cycle between both conditions indicating that the number of IAHC cells did not increase by proliferation upon Dll4 blockage (Fig 5h). This conclusion was experimentally supported by demonstrating similar levels of BrdU incorporation after IgG and $\alpha$ DII4 treatment (Suppl Fig 6a).

\section{Recruitment of new cells into the IAHC is controlled by DII4}

Having established that increased proliferation is unlikely to account for increased cluster sizes, our second hypothesis was that IAHC grow by recruitment of new cells into the already initiated IAHC. To test whether DII4 regulates the capacity of endothelial cells to be recruited into the nascent IACH, we used TAM-induced VeCadCre ${ }^{E R}$ :confetti embryos (Fig 6). We intracardiacally injected IgG or aDll4 followed by 5 hours incubation and processed the embryos for Kit staining ( $n=7$ per condition). We confirmed that transgenic modifications per se did not alter the total number nor the size of IAHCs and bigger clusters formed as a consequence of $\alpha$ DII4 blockage (Suppl Fig 7).

In these experiments, we observed more IAHC with colored cells in the aDll4-treated condition compared to IgG (Fig 6a; Fig 6c). This increased frequency is not associated with enhanced recombination in the endothelium (Fig 6d; Fig 6e) nor an increase in the total number of IAHC formed (Suppl Fig 7). Our interpretation is that recruitment events occur more often upon aDII4 treatment, leading to more IAHCs incorporating a colored cell. Consistently, the number of IAHC containing recombined cells per embryo is increased in these conditions (Fig6a; Fig6c). Collectively, these data indicate that $\alpha \mathrm{D} \| 4$ treatment increases the number of cells in IAHC by facilitating the chance of a colored cell to be incorporated in the growing cluster.

\section{Mathematical model supports a role for DII4 controlling cell recruitment in IAHC}


To better understand the parameters that control IAHC formation, we developed a probabilistic simulation of the process. In particular, we wanted to check what are the recruitment rates and cluster life-times that can explain the measured distributions of colored vs non-colored cells in IAHCs both in the IgG and $\alpha$ DII4 treated samples. The model assumes that clusters can be initiated with the same probability at any time point and can be detached after a certain time (defined as cluster life time). Since in physiological condition two-cell clusters are either all colored or all non-colored, we assume that the initiation stage always starts with two cells. Clusters in the model can then grow either by cell division events (assumed to happen every 11-13 hours, based on the BrdU analysis in Fig. 1F), or by recruitment events (see schematic in Fig. 7a). We focused on determining two parameters: (1) cluster life time and (2) average recruitment time (or recruitment probability). To determine the values of the parameters that best fit the measured IgG treated distribution, we performed 4000 simulations for each parameter set and checked whether the resulting distribution fit the experimental cluster sizes, and the fraction of colored cells in clusters (see details of the simulation in the methods). We found that values that best fit the measured IgG treated distribution are obtained for cluster life-time of 21 hours (90\% confidence interval $(\mathrm{Cl})$ 10-22 hours) and average recruitment time is 11 hours ( $90 \% \mathrm{Cl} 3-18$ hours) (Fig. 7b). We next simulated the case where samples were treated with $\alpha$ Dll4 for 5 hours. Here, we set the cluster life time to 21 hours (as obtained from the analysis of the IgG treated clusters) and checked what is the average recruitment time that can fit the observed data. We find that the best fit is obtained for recruitment time of 5 hours ( $90 \% \mathrm{Cl} 3-8$ hours), which is more than twice faster than the recruitment time without blocking DII4 (Fig. 7c). Using these parameters we run a simulation test for IgG and $\alpha$ DII4 treatment conditions, which resulted in a distribution of cluster size very similar to the one previously observed using empirical data (Fig 7d compared to Fig 6c).

There are several deviations between the cluster distributions observed in the simplified model proposed and the observed cluster distributions, potentially revealing additional processes affecting cluster sizes. First, some of the clusters observed in the $\alpha \mathrm{Dll} 4$ treated samples have more than 20 cells, significantly above the maximal cluster size obtained in our simulation. It is likely that such clusters arise due to effective merger of nearby clusters. 
Simulations performed assuming that the bigger clusters (>20 cells) are a result of a merger of two smaller clusters with half the size each, resulted in a similar estimation of the recruitment times (Suppl Fig. 8). Second, we do observe two two-cell clusters with one colored cell in the $\alpha \mathrm{Dll4}$ treated samples. This may suggest that recruitment times at the two-cell stage may be more significantly affected by the $\alpha$ DII4 treatment. Additional experiments may be required to resolve the recruitment times at different stages.

To further support the conclusion of the model that the increase in cluster size in $\alpha \mathrm{Dll} 4$ treated embryos is due to more frequent recruitment events, we imaged CD41:YFP ${ }^{+} ; \mathrm{H} 2 \mathrm{~B}-\mathrm{GFP}$ embryo organotypic slices. In these conditions we indeed identified cells adjacent to a CD41:YFP+ cluster getting incorporated into the cluster and turning on CD41:YFP expression

(Fig 7d and Movie 5). We also used organotypic slices from H2B-GFP embryo intracardiacally injected with $\mathrm{CD} 1^{549}$ antibody, and track the movement of a peripheral cell reaching the center of the close-by cluster (Movie 6). Overall, the confetti analysis, the mathematical modelling and the additional movies provide strong evidence that blocking of DII4 significantly enhances recruitment of cells into the IAHC.

\section{Recruitment of hemogenic $\mathrm{Gfi}^{+}$cells into the IAHC and progression to HSCs is restricted by DII4}

To functionally validate the effect of blocking DII4 in the hematopoietic activity of the IAHC cells, we performed 5h ex-vivo cultures of $\alpha$ DII4 treated and control AGM to test them for HSC and progenitor activity (Fig 8a). We dissected AGMs from IgG or aDll4 injected embryos, and after 48 hours we analyzed CFC activity (Fig 8B). We did not observe major changes in the number and quality of CFC, but a slight increase in the CFC-GM category after DII4 blockage (Fig 8b). Next, we tested whether $\alpha$ DII4 treatment was directly affecting the number or quality of hemogenic cells. To identify hemogenic cells, we took advantage of the Gfi1:tomato reporter mouse model which labels early hemogenic cells that are not yet expressing Kit or CD45. We found that Gfi1+ cells (hemogenic endothelial precursors) from early embryos (3032sp) were especially responsive to Dll4 blockage as they significantly transformed into Kit+CD45+ cells when compared with control-treated littermates after 5 hours treatment (Fig 
$8 c, 8 d$ and 8e). These results indicate that Dll4 signaling restricts the incorporation of hemogenic cells into the IAHCs.

Finally, we performed transplantation experiments to determine the effect of $\alpha \mathrm{Dll} 4$ treatment in HSC activity. AGM explants obtained from 30-34 sp CD45.2 embryos were injected with IgG or Dll4 blocker and then processed for hematopoietic transplantation together with 50,000 bone marrow CD45.1/2 support cells into CD45.1 lethally irradiated recipients (Fig 8a). Mice were monthly analysed for the presence of donor CD45.2 cells in the peripheral blood and 4 months after transplantation they were euthanized. We found that 5 of 12 mice transplanted with aDll4 treated AGM cells were highly reconstituted compared with only 2 of 8 mice transplanted with IgG control AGM (Fig 8f). Donor cells contributed to all different lineages in a similar way, but a higher contribution to the B-cell lineage from the $\alpha$ Dll4-treated cells was observed (Suppl Fig 9). Importantly, Lin-sca1+Kit+ (LSK) progenitors and CD150+CD48- HSCs were similarly preserved in the aDll4-treated engraftments (Fig 8g). Altogether these data strongly suggest that $\alpha \mathrm{D} \| 4$-treatment enhances the presence of engraftable LT-HSC without compromising their stemness.

In summary, our results indicate that at initial stages of IAHC formation, DII4 is restricting the recruitment of Gfi1+ hemogenic cells into the cluster. Once the cluster reaches three-four cells size, Dll4 is decreased and new Gfi1+ hemogenic cells can move into the IAHC (model in Fig 8h).

\section{DISCUSSION}

In this study, through a detailed characterization of the earliest stages of hematopoietic cluster formation, we identify a new role for the Notch-DII4 axis in regulating the origin of HSC. The emergence of the first definitive HSCs is a complex process requiring formation of specialized structures in the AGM that function as a supportive environment. During mammalian development, the appearance of HSCs correlates with the formation of IAHCS, which expose progenitors to the required set of molecular signaling for their specification and maturation. Despite the fact that IAHCs play an essential role in early hematopoiesis of higher 
vertebrates (reptiles, avian and mammals), little is known on how they generate and develop. Here, we describe for the first time, the two-step process of the formation of hematopoietic clusters, with an initial monoclonal phase as a result of one or two cell divisions, followed by a recruitment phase that contributes -together with proliferation- to cluster growth. We now provide data on cell cycle kinetics of the cluster that agrees with previous reports ${ }^{12}$ but the focus of the current investigations is on the impact of proliferation on IAHC clonality and architecture. Although polyclonality of IAHC have been suggested ${ }^{5,11}$, we now provide evidence for an initial monoclonal origin and the mechanism on how the polyclonality of larger IAHC is achieved and regulated by Notch-DII4 signals.

The Notch ligand DIl4 has a crucial role in arterial development, which limited genetic studies on aortic hematopoiesis due to the lack of specificity in targeting cluster cells. We now found that DII4 is expressed in the whole IAHC in small clusters (2-4 cells), but its expression is restricted to few cells in larger IAHC. This pattern suggests a defined function at different phases of IAHC growth that was addressed by testing the effect of $\alpha$ Dll4 blocking antibodies in the embryonic aorta. When DII4 is blocked, IAHC size increases dramatically from newly recruited neighboring cells, as shown by three independent approaches: Imaging of the confetti system shows increased heterogeneity in the mature clusters, time-lapse analyses of CD41:YFP ${ }^{+}$cells being incorporated into the IAHC and changes in the percentage of Gfi1+ hemogenic cells. These results indicate that DII4 might block the recruitment events until the IAHC core is completed. Once the initial cluster unit has been formed, few cells in the IAHC downregulate their levels of DII4 and the break is lifted, allowing recruitment to happen and consequently the cluster to grow. Our results show that the total number of IAHC is not increased after DII4 blockage, indicating that the emergence of new IAHC is not dependent on DII4-signalling. Instead, cells in already initiated IAHC (2-4 cells) express high levels of DII4 and prevent the entrance of new cells until Dll4 is downregulated.

The mechanism of cell recruitment in IAHC is reminiscent of the tip-stalk cell communication in angiogenic sprouting. Tip cells contain high DIl4 that signals to activate Notch in stalk cells and maintain the stalk phenotype. Jagged1 is present in the stalk cells and antagonizes DII4 activation of Notch ${ }^{27}$ while Fringe will potentiate the action of DII4. In the IAHC, DII4 is 
expressed in small clusters (similar to tip cells) and should signal to the endothelial cells preventing their inclusion into the cluster (similar to stalk cells). When Jag1 counteracts DII4 signal, either by fringe expression or by cis-inhibition (reviewed in ${ }^{28}$ ) Notch signal is inhibited and cells can move into the cluster. The role of fringe in cluster formation has not been explored yet, although expression of Manic fringe is observed in RNA-seq and immunostaining experiments (data not shown). The presence of fringe may inhibit Notch activation by Jag1 and allow DII4 signaling until the cells reach the cluster.

The specific function(s) of Notch in HSC development is not well understood. While reported data agrees that the level of Notch activity needs to be lower or inhibited from the endothelial cells to generate HSCs ${ }^{29-33}$, it is also well established that transcriptional Notch activity is required for this process ${ }^{22,32-34}$. However, the target cell(s) for Notch activation is unknown. We now show that hemogenic cells (Gfi1+Kit-CD41-CD45-) are one of the targets for DIl4 signal while maintained in the endothelium. When DII4 is reduced, cells can proceed to the IAHC where HSC can be generated. Our interpretation is that several Notch signals are required for HSC development, likely coming from different ligands (Jag1 and DII4) ${ }^{23}$, but also from different Notch receptors (Notch1 and Notch2) ${ }^{14}$.

Experiments using the $\mathrm{Gfil}^{+}$reporters support that cells recruited to the IAHC are hemogenic. The expression of Gfi1 identifies the first events of hemogenic conversion before the expression of Kit and CD45 which will be acquired in the HSC population ${ }^{10}$. Our results show that $\alpha$ DII4 treatment decreases the number of $\mathrm{Gfi1}^{+}$Kit- hemogenic cells and induces the formation of Gfi1+Kit+. This conversion is very robust when using 30-32 somite pair embryos strongly suggesting that this mechanism is crucial in the initial phases of definitive HSC development. Moreover, functional experiments testing HSC activity indicate that blocking aDII4 antibodies increase the engraftment potential of the early hemogenic cell, additionally suggesting that IAHC of bigger size contain the most mature HSC. Multilineage engraftment is observed from the aDll4-treated HSCs, although we find a higher potential for B-cell repopulation and surprisingly T-cell potential is unaffected, which will be a future matter of investigation. Single cell RNA sequencing data also confirms that the nature of Kit+ cells is 
unchanged at a transcriptomic level and altogether indicates that blocking DII4 does not disturb cell fate of HSCs, but it favors its generation or maturation.

Based on our imaging and clonality data, we developed a probabilistic simulation of IAHC formation. The observed distribution of colored and non-colored cells in IAHCs are captured in the model by assuming finite cluster life time of $\sim 21$ hours and an average recruitment time of 11 hours. Furthermore, the observed distributions in the $\alpha$ DII4 treated embryo can be best fitted to a recruitment time of only $\sim 5$ hours. We do note that we have observed a few clusters in the $\alpha$ DII4 treated embryos with $>30$ cells. Such large clusters are unlikely to arise from single recruitment events and indeed do not occur in the simulation. It may be possible that such large clusters emerge from fusion of smaller clusters. Hence, our quantitative modeling approach can test whether the observed IAHC formation can be explained by a combination of cell divisions, recruitment events and cluster detachments, and provide insights into the specific timescales associated with these processes. Moreover, our model can be used to determine the effect of physiological signals (e.g. Notch signaling) on these rates and hence helps understanding the molecular factors that affect HSC generation.

\section{MATERIAL AND METHODS}

\section{Animals}

C57BL/6 J wild-type, (Charles River Laboratories), CD41:YFP ${ }^{\text {tg/tg }}{ }^{18}, \mathrm{H} 2 \mathrm{~B}-\mathrm{GFP}{ }^{\text {tg/tg } 19}$ VeCadCre ${ }^{\text {ERT }}$ ${ }^{35}$ and R26R-Confetti ${ }^{36}$; Gfi1:tomato ${ }^{10}$ transgenic lines were used. Animals were kept under pathogen-free conditions, and all procedures were approved by the Animal Care Committee of the Parc de Recerca Biomedica de Barcelona, Licence number 9309 approved by the Generalitat de Catalunya. Embryos were obtained from timed pregnant females and staged by somite counting: E10.5 (32-38 sp). The detection of the vaginal plug was designated as day 0.5. Induction of recombination for the VeCadherinCre ${ }^{\mathrm{ERT}}: \mathrm{R} 26 \mathrm{R}-\mathrm{Confetti}$ line was performed injecting 50mg/Kg Tamoxifen (Sigma) into the pregnant female at E7.5 or E8.5. Cumulative intraperitoneal injections of $50 \mathrm{mg} / \mathrm{Kg}$ BrdU was injected at different time point for kinetic analysis as previously described ${ }^{20}$. Pregnant females were euthanized after $1 \mathrm{~h}$ BrdU injection 
and embryo processed for immunostaining. For cumulative analyses pregnant females received BrdU every 2 hours and then euthanized after 4h, 6h and 8hours for embryo collection and processing.

\section{DII4-blocking and embryo culture}

C57BL/6 J wild-type or VeCadherinCre ${ }^{\text {ERT }}:$ R26R-Confetti embryos were collected at E10.5 and placed in sterile PBS 10\% inactivated fetal bovine serum (FBS) for placenta, yolk sac and tail removal. Blocking antibody $\square$ Dll4 (Genentech) or mock antibody IgG (Goat Anti- Human Ig, Southern Biotech 2010-01) at $1 \mu \mathrm{g} \mathrm{ml}^{-1}$ were slowly injected intracardiacally until complete clearance of the aortic blood. Treated embryos were then incubated for additional 5hours at $37^{\circ} \mathrm{C}$ in a humidified atmosphere with $5 \% \mathrm{CO}_{2}$ in complete myeloid long-term medium (STEMCELL Technologies - supplemented with $10 \mathrm{ng} \mathrm{ml}^{-1}$ interleukin (IL)-3 and $10 \mu \mathrm{M}$ hydrocortisone (Sigma-Aldrich) containing $5 \mu \mathrm{g} \mathrm{ml}^{-1}$ of the relevant antibody. For BrdUincorporation analysis intraperitoneal injections of $50 \mathrm{mg} / \mathrm{Kg}$ BrdU was injected once to the pregnant female and embryos processed as above.

\section{Organotypic slice culture and Time-lapse imaging and embryonic culture}

CD41:YFP ${ }^{\text {tg/tg }}$ crossed with H2B-GFP ${ }^{\text {tg/tg }}$ embryos were collected at E10.5. Embryos were placed in sterile PBS 10\% FBS for placenta and yolk sack removal. For direct staining of aortic endothelium $5 \mathrm{ul}$ of $\mathrm{CD} 31^{594}$ directly conjugated antibody (BD Bioscience) was injected into the beating heart and whole embryos were incubated in ice for $20 \mathrm{~min}$. Embryos were then sectioned using a Maclllwain tissue chopper to obtain horizontal slices of 100um thickness. Organotypic slices were then transferred onto an O-ring chamber and trapped in Agarose 1\% (Sigma-Aldrich). Slices were kept in myeloid long-term medium (STEMCELL Technologies supplemented with $10 \mathrm{ng} \mathrm{ml}^{-1}$ interleukin (IL)-3 and $10 \mu \mathrm{M}$ hydrocortisone (Sigma-Aldrich) in a humidified chamber with $5 \% \mathrm{CO}_{2}$ at $37^{\circ} \mathrm{C}$ for the whole duration of the live imaging. Time Lapse was performed using an inverted Leica-SP5 confocal with excitation lasers 488, 514 and 549 wavelenght as previously described ${ }^{4}$.

Immunostaining 
For tissue-section immunostainings, embryos were fixed $1 \mathrm{~h}$ in $4 \%$ paraformaldehyde (SigmaAldrich) at $4{ }^{\circ} \mathrm{C}$, included in Optimal Cutting Temperature (OCT) (Tissue-Tek, Sakura) and sectioned at $25 \mu \mathrm{m}$. Permeabilization was performed with 0,5\% triton for $30 \mathrm{~min}$ at RT and blocking step was done with a solution of $10 \%$ FBS and 0,1\%triton for 1 hour at RT. Primary antibodies were used at the following concentrations: anti-cKit 1:100 (rat, BD); anti-pH3 1:300 (rabbit, Upstate Cell Signaling ); anti-GFP 1:300 (rabbit, Molecular probes); anti-Jag1 1:400 (sc6011-Santa Cruz), anti-DII4 1:200 (goat, R\&D AF1389), anti-DII4 1:1000 (human, Genentech) anti-ICN1 1:100 ( $\alpha$-N1Icv monoclonal antibody Cell signaling \#4147S); anti-BrdU 1:50 (ms, BD Bioscience); Ki67 1:100 (ms, Novacastra); biotinylated anti-CD31 1:100 (BD 553371).

For mouse antibodies, slices were additionally incubated with FAB 1:10 (Roche) in PBS $2 \mathrm{~h}$ at RT.

For CD31 staining, an additional blocking of endogenous biotin was performed (Endogenous Avidin+Biotin Blocking System, ab3387 abcam) and detected with streptavidin Alexa Red 555 (BD 32355).

For BrdU and Ki67 staining a primary antigen retrieval was performed in $10 \mathrm{mM}$ sodium citrate $\mathrm{pH} 6,30 \mathrm{~min}$ at $80^{\circ} \mathrm{C}$. For BrdU staining an additional incubation in $\mathrm{HCl} 2 \mathrm{~N}$ for 20 min was performed. Fluorochrome-conjugated (1:1000 Alexa Fluor 488,546,647) or horseradish peroxidase (HRP)-conjugated (1:200, Dako) secondary antibodies were used for detection. Three embryos per groups were analysed at each time point and positivity on total number of 4,6-diamidino-2-phenylindole (DAPI; Invitrogen)-positive nuclei counted inside the clusters.

Whole-mount immunostaining was performed as described ${ }^{3}$. Briefly, embryos were fixed for $20 \mathrm{~min}$ in $2 \%$ paraformaldehyde, dehydrated in methanol and trimmed. Primary antibodies were used at the following concentration: anti-cKit 1:100 (rat, BD Bioscience), and biotinylated anti-CD31 1:100 (BD 553371). Detection was achieved with horseradish peroxidase (HRP)-conjugated secondary antibody (1:200, Dako) developed with tyramide amplification system TSA (PerkinElmer) and streptavidin Alexa Red 555 (BD 32355).

To analyze the IAHC at a rostro-caudal level, a range of $250-1500 \mu \mathrm{m}$ of aortic endothelium was sectioned and immunostained with the kit antibody. Section reconstruction from confocal 
images was performed and the number of $\mathrm{Kit}^{+}$cells and IAHC was quantified in individual aortas. IAHCs were classified: 2-5 cells small cluster; 6-10 cells medium cluster; $11-15$ cells large cluster; above 16 cells very large cluster.

\section{Equipment and settings}

All immunofluorescence images were produced using a Confocal Leica SP5 or SP8 with the following settings: $1024 \times 1024$ pixel dimension; $400 \mathrm{~Hz}$ laser; frame average 3 or 4 . photomultipliers PMT or Hyd. Fluorochromes used: Alexa 488,546,594,647 or CFP,RFP,YFP,GFP excitation for Confetti analyses. A range of $25 \mu \mathrm{m}$ (standard) to $70 \mu \mathrm{m}$ (whole mount) thick Z-stack was analyzed collecting images every $2.5 \mu \mathrm{m}$ (standard) or $10 \mu \mathrm{m}$ (whole mount). Images were processed using Imaris 7.0 and 8.0 software, Bitplane. Gaussian smoothing was applied to all channels when needed and equally to all images of the same batch. For Time-lapse videos a Leica SP5 inverted confocal microscope equipped with CO2 and temperature controlled chamber $\left(\mathrm{CO} 2=5 \%, \mathrm{~T}=37^{\circ} \mathrm{C}\right)$ was used. Laser scanned the samples every $15-19 \mathrm{~min}$ at $700 \mathrm{~Hz}$ for a maximal time of 9 hours. Movies were processed post-imaging with ImageJ-Fiji to correct drifting (ImageJ-Drift Correction) and recorded in Imaris 8.0 (Bitplane) for further analysis and automatic tracking. Tracking analyses over time was performed using the spot function and central point automatic tracking in Imaris.

\section{Haematopoietic progenitor assay}

C57BL/6 J wild-type embryos at 32-35sp were collected and intracardiacally injected with control IgG or blocking antibody $\alpha \mathrm{Dll} 4$ as described above. After $5 \mathrm{~h}$ incubation AGMs were incubated for $20 \mathrm{~min}$ at $37^{\circ} \mathrm{C}$ in $0.12 \%$ collagenase (Sigma-Aldrich). Single cell suspension was obtained and seeded in quadruplicates in Methocult M-3434 semi-solid medium (Stem Cell Technologies) at $37^{\circ} \mathrm{C}$ with $5 \% \mathrm{CO}_{2}$. Colony-forming units were counted after 7 days ( $\mathrm{n}=3$ embryos/group).

Fluorescence-activated cell sorter (FACS) analysis and purification

AGMs were harvested and dissociated in single-cell suspension. Cells were then washed with PBS $+10 \%$ FBS before antibody staining. Antibody staining was performed in PBS 
supplemented with 10\% FCS in the dark, at room temperature for $15 \mathrm{~min}$, or carried out on ice for 30 min. CD117 (c-kit; APC-Cy7 or APC-eFluor780), CD31 (APC or FITC), CD45.1 (APC-Cy7) and CD45.2 (FITC), Dll4 (PerCP5 or APC) Jag1 (PE or FITC), Ter119 (Pe-Cy7) Ki67 (APC) antibodies were purchased from BD (Franklin Lakes, NJ, USA). DAPI (4,6-diamidino-2phenylindole; D1306, Invitrogen, Waltham, MA, USA) was used for viability. FACS was performed on LSRII (BD), sorting on FACSAria (BD) and data were analyzed with FlowJo v.10 (TreeStar, Inc., Ashland, OR, USA).

\section{AGM explants and Transplantation experiments}

Charles River C57BL/6 (CD45.2) WT embryos of 32-34sp were treated as described above injecting intracardiacally $\alpha$ DII4 or anti-IgG. AGM were then dissected and cultured as explant as previously described ${ }^{37}$. In brief, AGMs were deposited on nylon filters (Millipore) placed on metallic supports and cultured in myeloid long-term culture medium (Stem Cell Technologies) supplemented with $10 \mu \mathrm{M}$ hydrocortisone (Sigma-Aldrich). After 24hours the explanted AGMs were digested in $0.1 \%$ collagenase (Sigma-Aldrich) in PBS supplemented with 10\% FBS for 20 min at $37^{\circ} \mathrm{C}$ and used for cell transplantation. Donor cells (CD45.2) were transplanted together with 200000 BM support cells (CD45.1) into lethally irradiated (8 Gy) recipients (CD45.1). Peripheral blood (PB) donor chimerism was analyzed by FACS at 4, 10-12 and 16 weeks. Lineage analysis on BM-derived cells was performed at 16 weeks post-transplant by flow cytometry with specific antibodies: CD3, B220, Ter119, Mac1, and Gr1 (mouse lineage panel; BD).

\section{Bulk RNA sequencing and analysis}

For RNA-seq study, the SMARTer UltraLow RNA kit v.4 for Illumina Sequencing was used. Briefly, $\alpha \mathrm{DI} / 4$ and control-treated AGM were digested with $0.1 \%$ collagenase and single-cell suspension was stained as described. Different subpopulations were directly sorted in a total volume of $10 \mu \mathrm{l}$ of reaction buffer and processed for obtaining cDNA following manufacture's protocol. cDNA amplification was performed by Long Distance PCR (LD-PCR) and the PCRamplified cDNA purified by immobilization on AMPure XP beads (Agencourt AMPure XP kit). Samples were analyzed with Agilent High Sensitivity DNA Kit (Agilent, Cat. No. 5067-4626). 
Covaris shearing was used for Illumina Low input sample preparation and Double last bead purification was performed to remove fragments below $200 \mathrm{bp}$. Next, samples were used to generate an Illumina sequencing library by NEBNext Ultra protocol following kit instructions. After PCR amplification of the library, the quality was checked on a Bioanalyzer and total cDNA was sequenced using an Illumina HiSeq 2000 sequencer.

MultiQC tool (Ewels et al. 2016) implementing QualiMAP (Quality control), STAR (RNA-seq aligner) and DESeq2 (gene differential expression) packages, was run to obtain normalized gene counts and asses differential gene expression levels among three cell populations Kp45p, Kp45m and Km41p. To test for statistically significant differences between populations, a 1way ANOVA test was performed ( $p$-value< 0.001). This resulted in three sets of up- and down-regulated genes; intersecting these allowed discriminating the differentially expressed genes (DEGs) exclusive to each the cell population. Principal Component Analysis (PCA) was then performed using the 24 samples as observations and 2770 genes (the total number of DEGs) as variables. To identify DEGs in control and treated populations, we performed 1-way ANOVA test using high p-value cutoff (0.05). A PCA analysis using 21 samples as observations (3 samples with PC1 score values $\approx 0$ were excluded) and 370 DEGs indicated a clear-cut discrimination according to treatment with antibody. To identify DEGs between treated samples and controls, considering those DEGs among phenotypes, a 2-way ANOVA test was performed ( $p$-value < 0.05). Gene Ontology categories corresponding to the 6 resulting genes sets were interrogated using the DAVID Database (Huang et al. 2007) for the functional enrichment analysis. Only functional categories from Biological Processes annotations were considered, filtering out those that involved less than 10 genes.

\section{Single cell RNA Seq}

Cells were sorted in $2.3 \mu$ l of lysis buffer containing 0.2\% Triton X-100 (Sigma-Aldrich) and $1 U$ of Superase-In RNase Inhibitor (Ambion) and processed following the Smart-Seq2 protocol $\left({ }^{26}\right)$. Following preamplification, all samples were purified using Ampure XP beads (Beckman Coulter) at a ratio of 1:0.6. The cDNA was then quantified using the Quant-iT PicoGreen dsDNA Assay Kit (Thermo Fisher) and size distributions were checked on high-sensitivity DNA chips (Agilent Bioanalyzer). Samples were used to construct Nextera XT libraries (Illumina) 
from 125 pg of preamplified cDNA. Libraries were purified and size selected (0.5X-0.7X) using Ampure XP beads. Then, libraries were quantified using KAPA qPCR quantification kit (KAPA Biosystems) and sequenced in a Illumina HiSeq 4000 instrument.

Bioinformatic Analysis of sc RNA-seq

Reads were mapped to the Mus musculus genome (EMSEMBL GRCm38.p4 Release 81) and ERCC sequences using GSNAP (version 2014-10-07) with -B 5 (batch mode 5) -n 1 (maximum pathsallowed: 1) $-\mathrm{Q}$ (if maximum paths more than $\mathrm{n}$, not print) $-\mathrm{N} 1$ (look for novel splicing). HTseq-count was used to count reads mapped to each gene with -s no (non-strand specific mode) ${ }^{38}$ For further analyses, we only retained samples that had (1) more than 100,000 reads mapped to nuclear mRNAs; (2) a proportion of reads mapped to genes (nuclear + mitochondrial) relative to total reads above $20 \%$; and (3) less than $20 \%$ of mapped reads allocated to mitochondrial genes. Overall, 483 cells (84\%) passed our quality controls distributed as 239 cells from 4 control embryos and 244 cells from 4 a-Dll4 treated embryos.

Data were normalised for sequencing depth and RNA quantity using size factors calculated on endogenous genes ${ }^{39}$ ]). Highly variable genes were identified as described ${ }^{39}$ ), using a false discovery rate threshold equal to 0.1. Only highly variable genes were considered to perform tSNE analysis, using the Rtsne package from $R$.

Clusters were identified using ICGS in AltAnalyze package ${ }^{25}$. For integration with previously published dataset ${ }^{26}$, the data was downloaded from GEO repository (GSE67120) and processed as indicated above. Differentially expressed genes were obtained for endothelial cells, pre-HSCs (T1 and T2), HSCs (E12 and E14) by comparing each group with the rest of the dataset. The union of the top 50 differentially expressed genes (log2 fold change $>2$, adjusted $\mathrm{p}$ value $(\mathrm{BH}$ method) $<0.1$ and baseMean $>50)$ from each comparison was selected to perform hierarchical clustering in our dataset using 'ward.D2' as the agglomeration method.

Cell cycle stage was predicted using the function cyclone in $\mathrm{R}$ package scran ${ }^{40}$. Differential expression analysis was performed using the package DESeq2 (version 1.18.1) ${ }^{41}$.

Clonal analysis 
VeCadherinCre ${ }^{E R T}:$ R26R-Confetti analysis was performed using endogenous fluorescence of the transgene and additional staining for Kit or CD31. Distinction of the confetti colors was done under a confocal microscope using appropriate excitation length and filter combinations (Leica SP5 or SP8).

\section{Mathematical modelling}

We use a markov chain model to simulate the formation of the cluster. The simulation has two stages: 1 . Stage of cluster initiation. 2. Stage of cluster proliferation and recruitment of more cells from the endothelial tissue.

The simulation algorithm is as follows:

A time step loop is started from time 0 until some $T_{\text {lif e time }}$ which we define as the cluster life time. This is the time after which a cluster is released from the aorta and can no longer be captured in the measurement. Assuming that cluster initiation is a random event, clusters can be initiated any time between 0 and $T_{\text {life }}$ time such that the probability per unit time for cluster initiation is uniform and is given by $1 / T_{\text {life time }}$. Since in physiological conditions all two-cell clusters we observed experimentally are either all colored or all non-colored we assume that the initiation stage always starts with two cells. After an initiation event has occurred the simulation moves on to the cluster proliferation and cell recruitment stage.

In the proliferation and cell recruitment stage there are two possible occurrences in each time step:

1. Each cell in the cluster can divide. Since we observed an average cell cycle time of 12 hours, we assumed that cell division can occur between 11-13 hours after the last division event. The probability of division increases linearly between 11-13 hours such that the probability of division at 10 hours after the last division is 0 and the probability of division 13 hours after the last division is 1.

2. In each time step a recruitment event can occur with probability per unit time of $p_{\text {recruitment }}=\frac{1}{T_{\text {recruitment }}}$ (with units of hour $\left.^{-1}\right)$. The color of the recruited cell goes 
according to the percent of colored cells in the endothelial tissue such that the probability per unit time of recruitment of a colored cell is $p_{\text {recruitment colored }}=$ $p_{\text {recruitment }} *$ percent of colored cells in the tissue and the probability per unit time of recruitment of a non-colored cell is $p_{\text {recruitmetn colored }}=p_{\text {recruitment }} *$ percent of non-colored cells in the tissue. After a cell is recruited, it is assumed that it has another cell cycle of 11-13 hours before the next division event.

There are two unknown parameters determining the formation of the cluster: $T_{\text {life }}$ time and $p_{\text {recruitment }}$. First, we determined what are the best $T_{\text {life time }}$ and $p_{\text {recruitment }}$ that fit the data of the IgG treated cells. We ran the simulation 4000 times for each combination of the two parameters. From the simulations of each set of parameters, we fitted the cluster size and ratio of colored cells in the cluster to the measured data (Average experimental cluster size $=$

$5.5 \pm 2.9$; Average experimental ratio of colored cells $=0.54 \pm 0.38$ ). The fit was done using a Hotelling's T2 test. The best fitting $T_{\text {life time }}$ and $p_{\text {recruitment }}$ we extracted are $T_{\text {life time }}=21, p_{\text {recruitment }}=1 / 11$.

Next, we determined the effect of $\alpha$ Dll4 treated on $p_{\text {recruitment }}$. The simulation for the Dll4 treated clusters is as follows:

1. Initially, the clusters develop without the effect of $\alpha \mathrm{D}$ ll4. The simulation is run according to the parameters determined in the IgG treated cells as described above. This is the stage of cluster formation before $\alpha$ DII4 treatment.

2. After a cluster is initiated, it continues to develop for a period of $T_{\text {life }}$ time, however this time it assumed that it is treated with $\alpha D \| 4$ in the final 5 hours of the simulation. During the $\alpha \mathrm{DI} / 4$ treatment the probability per unit time of recruitment is assumed to be $p_{\text {recruitment } \alpha D l l 4}=\frac{1}{T_{\text {recruitment } \alpha D l l 4}}$.

We determined the $p_{\text {recruitment } \alpha \text { dll4 }}$ that fits the measured data of $\alpha$-Dll4 treated clusters the best. We ran the simulation 4000 for each possible $p_{\text {recruitment } \alpha d l l 4}$, and again fitted the cluster size and ratio of colored cells in the cluster to the measured data (Average 
experimental cluster size $\alpha$ DII4-treated cells= 7.1 \pm 6.7 ; Average experimental ratio of colored cells $=0.50 \pm 0.37)$.

The Matlab code for the stochastic simulation is available at https://github.com/OhadGolan/ClusterFormation.

\section{Statistical analyses}

Statistical comparison between two or more groups was performed using an unpaired, Student's T-Test with equal variance. For the mathematical model the Hotelling's T-Squared test multivariate counterpart of the T-Test was used ${ }^{42}$. For comparing distribution of clusters in size categories the non-parametric Mann-Whitney U Test was applied.

\section{AUTHOR CONTRIBUTION}

C.P. designed and performed most experiments, analyzed the data and wrote the manuscript, O.G, F.J.C.N, R.T. and CRH designed and performed experiments and analyzed the data. F.C., J.G., S.J.K., R.S., S.A.M. and A.M. performed experiments. X.W., Y.G. and PC performed bioinformatics analysis. E.D., L.E., B.G. and D.S. designed and supervised experiments and data and contributed with valuable tools. A.B. designed and supervised the research project, analyzed the data, and wrote the manuscript.

\section{ACKNOWLEDGEMENTS}

We thank Thomas Graf (CRG), Minhong Yan (Genentech), Georges Lacaud (CRUK Manchester Institut) and Ralf Adams (Max Planck Institute for Molecular Medicine, Münster) for important mice models and reagents. Thanks to Catherine Robin, Timo Zimmermannand Raul GómezRiera for imaging advice. Thanks to the PRBB facilities: animal facility, flow cytometry, genomics, CNAG-CRG, confocal microscopy for critical technical assistance. Thanks to all the 
members of the lab for helpful critical discussions. This research was funded by the Ministerio de Economía y Competitividad (SAF2016 -75613-R) and Generalitat de Catalunya, Agència de Gestió d'Ajuds Universitaris i de Recerca (AGAUR) (2017 SGR 135) and PERIS-SLT002/16/00299 to AB. CP was a recipient of Juan de la Cierva fellowship (FJCI2014-19870), RT is a recipient of Beatriu de Pinos (2016 BP 00021), JG is a recipient of PERIS (SLT002/16/00070).

\section{REFERENCES}

1. Bertrand, J. Y. et al. Haematopoietic stem cells derive directly from aortic endothelium during development. Nature 464, 108-111 (2010).

2. Kissa, K. \& Herbomel, P. Blood stem cells emerge from aortic endothelium by a novel type of cell transition. Nature 464, 112-115 (2010).

3. Yokomizo, T. \& Dzierzak, E. Three-dimensional cartography of hematopoietic clusters in the vasculature of whole mouse embryos. Development 137, 3651-3661 (2010).

4. Boisset, J.-C. et al. In vivo imaging of haematopoietic cells emerging from the mouse aortic endothelium. Nature 464, 116-120 (2010).

5. Ganuza, M. et al. Lifelong haematopoiesis is established by hundreds of precursors throughout mammalian ontogeny. Nat. Cell Biol. 19, 1153-1163 (2017).

6. Kumaravelu, P. et al. Quantitative developmental anatomy of definitive haematopoietic stem cells/long-term repopulating units (HSC/RUs): role of the aorta-gonad-mesonephros $(A G M)$ region and the yolk sac in colonisation of the mouse embryonic liver. Development 129, 4891-4899 (2002).

7. Eich, C. et al. In vivo single cell analysis reveals Gata2 dynamics in cells transitioning to hematopoietic fate. J. Exp. Med. 215, 233-248 (2018). 
8. Gao, L. et al. RUNX1 and the endothelial origin of blood. Exp. Hematol. 68, 2-9 (2018).

9. Rybtsov, S. et al. Hierarchical organization and early hematopoietic specification of the developing HSC lineage in the AGM region. J. Exp. Med. 208, 1305-1315 (2011).

10. Thambyrajah, R. et al. GFI1 proteins orchestrate the emergence of haematopoietic stem cells through recruitment of LSD1. Nat. Cell Biol. 18, 21-32 (2016).

11. Zape, J. P., Lizama, C. O., Cautivo, K. M. \& Zovein, A. C. Cell cycle dynamics and complement expression distinguishes mature haematopoietic subsets arising from hemogenic endothelium. Cell Cycle 16, 1835-1847 (2017).

12. Batsivari, A. et al. Understanding Hematopoietic Stem Cell Development through Functional Correlation of Their Proliferative Status with the Intra-aortic Cluster Architecture. Stem Cell Reports 8, 1549-1562 (2017).

13. Dzierzak, E. \& Bigas, A. Blood Development: Hematopoietic Stem Cell Dependence and Independence. Cell Stem Cell 22, 639-651 (2018).

14. Souilhol, C. et al. Developing HSCs become Notch independent by the end of maturation in the AGM region. Blood 128, 1567-1577 (2016).

15. Krebs, L. T. et al. Notch signaling is essential for vascular morphogenesis in mice. Genes Dev. 14, 1343-1352 (2000).

16. Robert-Moreno, A. et al. Impaired embryonic haematopoiesis yet normal arterial development in the absence of the Notch ligand Jagged1. EMBO J. 27, 1886-1895 (2008).

17. Duarte, A. et al. Dosage-sensitive requirement for mouse DII4 in artery development. Genes Dev. 18, 2474-2478 (2004). 
18. Zhang, J. et al. CD41-YFP mice allow in vivo labeling of megakaryocytic cells and reveal a subset of platelets hyperreactive to thrombin stimulation. Exp. Hematol. 35, 490-499 (2007).

19. Hadjantonakis, A.-K. \& Papaioannou, V. E. Dynamic in vivo imaging and cell tracking using a histone fluorescent protein fusion in mice. BMC Biotechnol. 4, 33 (2004).

20. Nowakowski, R. S., Lewin, S. B. \& Miller, M. W. Bromodeoxyuridine immunohistochemical determination of the lengths of the cell cycle and the DNA-synthetic phase for an anatomically defined population. J. Neurocytol. 18, 311-318 (1989).

21. Kumano, K. et al. Notch1 but not Notch2 is essential for generating hematopoietic stem cells from endothelial cells. Immunity 18, 699-711 (2003).

22. Robert-Moreno, A., Espinosa, L., de la Pompa, J. L. \& Bigas, A. RBPjkappa-dependent Notch function regulates Gata2 and is essential for the formation of intra-embryonic hematopoietic cells. Development 132, 1117-1126 (2005).

23. Gama-Norton, L. et al. Notch signal strength controls cell fate in the haemogenic endothelium. Nat Commun 6, 8510 (2015).

24. Ridgway, J. et al. Inhibition of DII4 signalling inhibits tumour growth by deregulating angiogenesis. Nature 444, 1083-1087 (2006).

25. Olsson, A. et al. Single-cell analysis of mixed-lineage states leading to a binary cell fate choice. Nature 537, 698-702 (2016).

26. Zhou, F. et al. Tracing haematopoietic stem cell formation at single-cell resolution. Nature 533, 487-492 (2016).

27. Benedito, R. et al. The notch ligands Dll4 and Jagged1 have opposing effects on angiogenesis. Cell 137, 1124-1135 (2009). 
28. Bigas, A. \& Espinosa, L. The multiple usages of Notch signaling in development, cell differentiation and cancer. Curr. Opin. Cell Biol. 55, 1-7 (2018).

29. Lizama, C. O. et al. Repression of arterial genes in hemogenic endothelium is sufficient for haematopoietic fate acquisition. Nat Commun 6, 7739 (2015).

30. Zhang, C. et al. m6A modulates haematopoietic stem and progenitor cell specification. Nature 549, 273-276 (2017).

31. Zhang, P. et al. G protein-coupled receptor 183 facilitates endothelial-to-hematopoietic transition via Notch1 inhibition. Cell Res. 25, 1093-1107 (2015).

32. Richard, C. et al. Endothelio-mesenchymal interaction controls runx1 expression and modulates the notch pathway to initiate aortic hematopoiesis. Dev. Cell 24, 600-611 (2013).

33. Burns, C. E., Traver, D., Mayhall, E., Shepard, J. L. \& Zon, L. I. Hematopoietic stem cell fate is established by the Notch-Runx pathway. Genes Dev. 19, 2331-2342 (2005).

34. Guiu, J. et al. Hes repressors are essential regulators of hematopoietic stem cell development downstream of Notch signaling. J. Exp. Med. 210, 71-84 (2013).

35. Wang, Y. et al. Ephrin-B2 controls VEGF-induced angiogenesis and lymphangiogenesis. Nature 465, 483-486 (2010).

36. Snippert, H. J. et al. Intestinal crypt homeostasis results from neutral competition between symmetrically dividing Lgr5 stem cells. Cell 143, 134-144 (2010).

37. Medvinsky, A. \& Dzierzak, E. Definitive hematopoiesis is autonomously initiated by the AGM region. Cell 86, 897-906 (1996).

38. Anders, S., Pyl, P. T. \& Huber, W. HTSeq--a Python framework to work with highthroughput sequencing data. Bioinformatics 31, 166-169 (2015). 
39. Brennecke, P. et al. Accounting for technical noise in single-cell RNA-seq experiments. Nat. Methods 10, 1093-1095 (2013).

40. Lun, A. T. L., McCarthy, D. J. \& Marioni, J. C. A step-by-step workflow for low-level analysis of single-cell RNA-seq data with Bioconductor. F1000Res 5, 2122 (2016).

41. Love, M. I., Huber, W. \& Anders, S. Moderated estimation of fold change and dispersion for RNA-seq data with DESeq2. Genome Biol. 15, 550 (2014).

42. Sánchez, J. MARDIA, K. V., J. T. KENT, J. M. BIBBY: Multivariate Analysis. Academic Press, London-New York-Toronto-Sydney-San Francisco 1979. xv, 518 pp., \$61.00. Biometrical Journal 24, 502-502 (1982). 


\section{Figure 1}

a

(i) bulging cell

(ii) dividing cell

(iii) cluster core
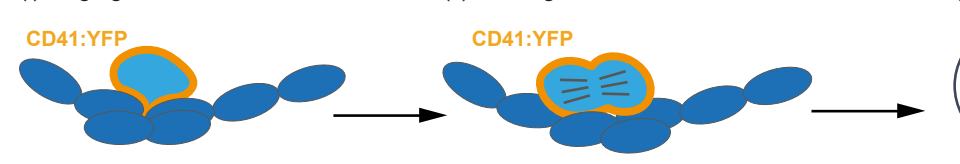

b

ventral endothelium

ventral endothelium
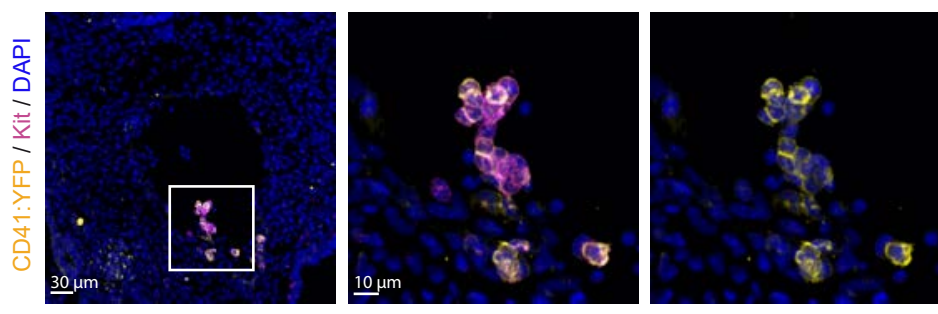

ventral endothelium

C
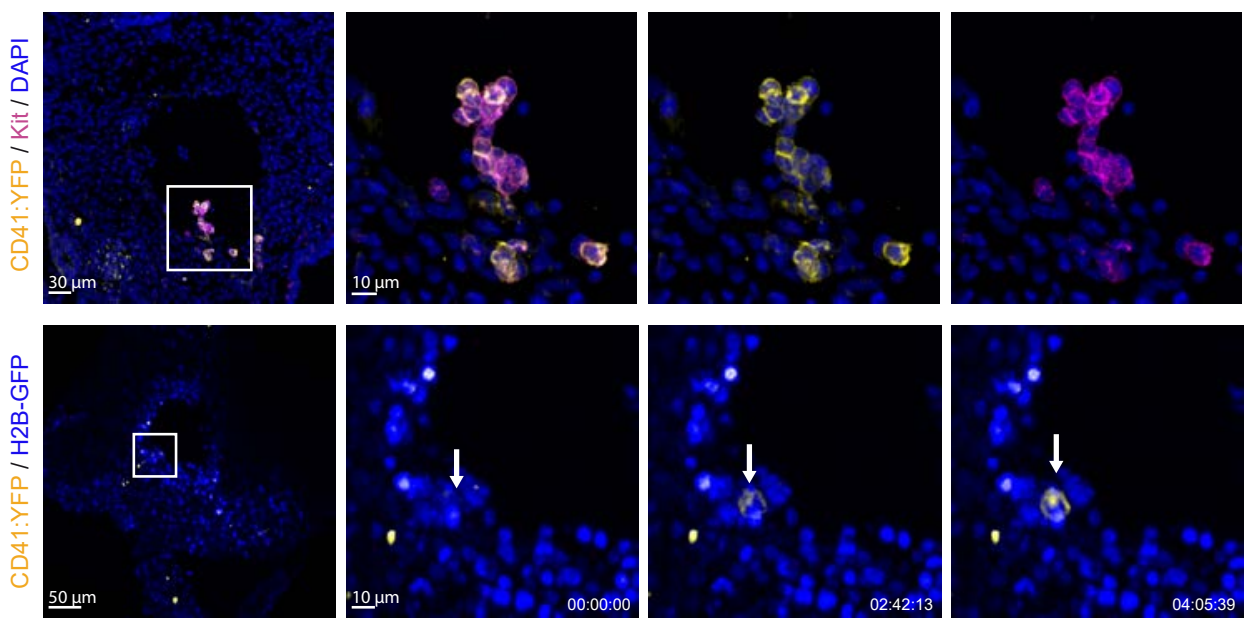

d
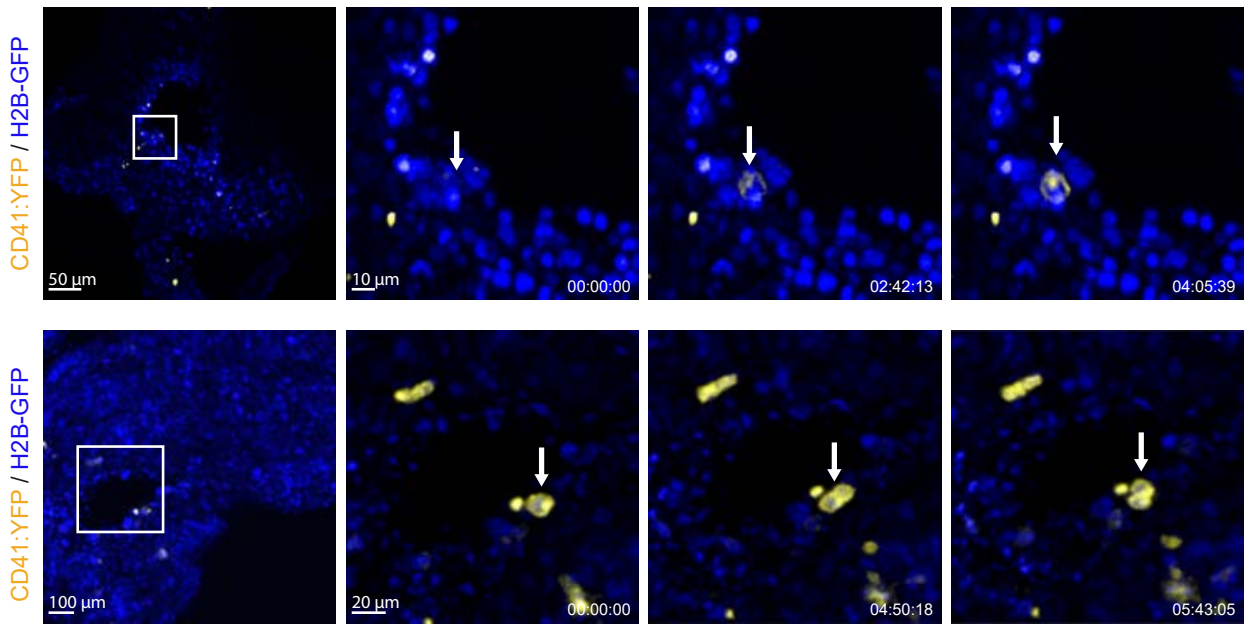

e
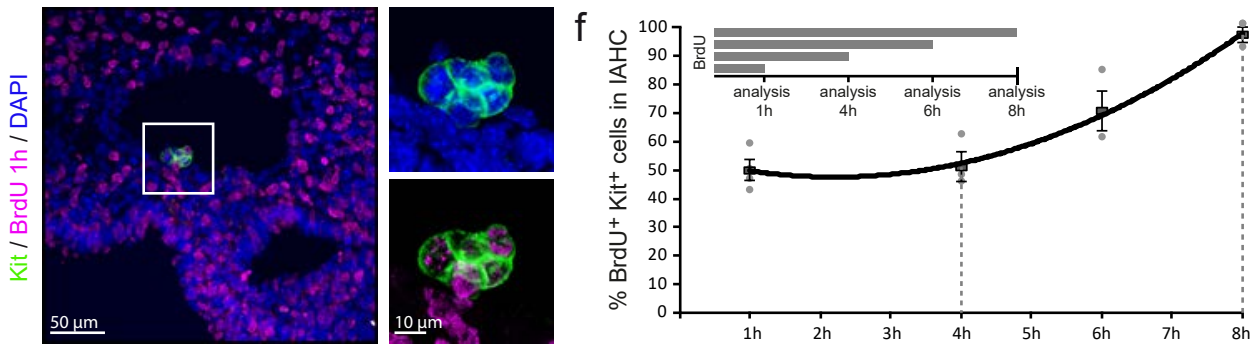
Figure 1: Bulging cells divide to form the monoclonal core of the IAHC (a) Schematic representation of initial events of IAHC formation. (i) A single cell protrudes into the lumen of the aorta and starts expressing CD41. (ii) The CD41:YFP+ bulging cell undergoes mitosis (iii), forming the core of the IAHC. (b) Expression of Kit (magenta) largely overlaps with the expression of CD41:YFP (yellow) in IAHC. Multistack reconstruction of confocal images. Scale bars: $30 \mu \mathrm{m}$ overview, 10 $\mu \mathrm{m}$ magnification. (c) Snapshots of Movie 1. Time-lapse of embryonic organotypic slice culture from CD41:YFP (yellow): H2BGFP (blue) reporter mouse shows the emergence of a single cell protruding from the endothelial layer

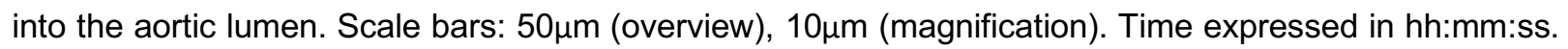
(d) Snapshots of Movie 2. Time-lapse of embryonic organotypic slice culture from CD41:YFP (yellow): H2B-GFP (blue) reporter mouse shows a single CD41:YFP expressing cell that divides once on the aortic endothelium. Scale bars: $100 \mu \mathrm{m}$ (overview), $20 \mu \mathrm{m}$ (magnification). Time expressed in hh:mm:ss. (e) BrdU incorporation in the Kit+ IAHC upon cumulative administration. Multistack reconstruction of confocal images. Representative picture of a short BrdU pulse (1h). Scale bars: $50 \mu \mathrm{m}$ (overview), 10 $\mu \mathrm{m}$ (magnification). (f) Quantification of the percentage of Kit+ cells within IAHCs incorporating BrdU using confocal images. All cells (Labelling Index $=100 \%$ ) can be labelled after $8 \mathrm{~h}$, indicating the lack of quiescent cells inside the IAHCs. Graph represents mean \pm SE. 


\section{Figure 2}

a

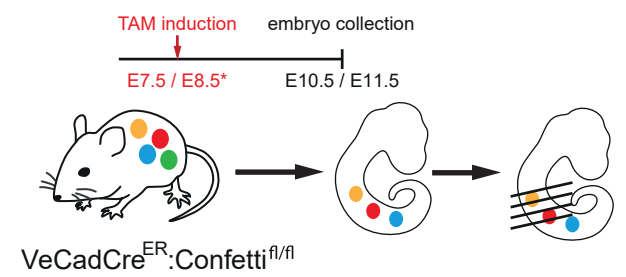

C

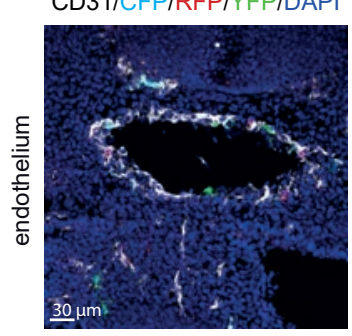

d

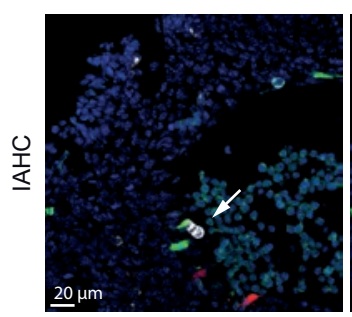

CFP / RFP / YFP

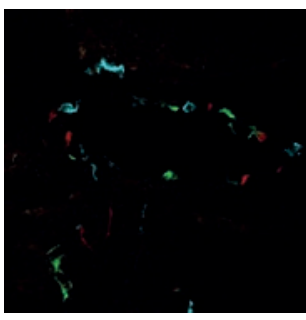

CFP / RFP / YFP / DAPI

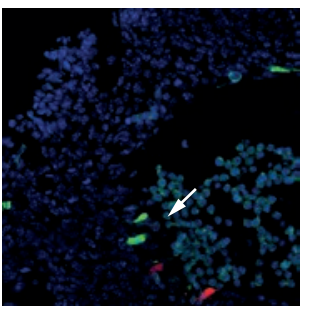

b

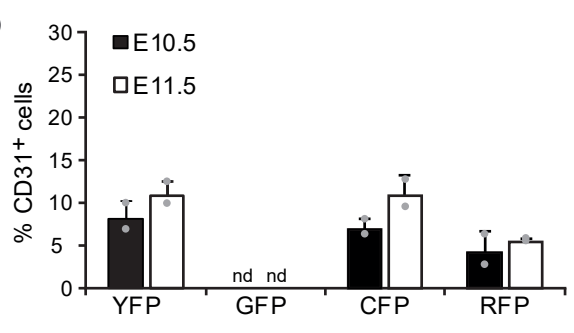

CD31 / CFP / RFP / YFP

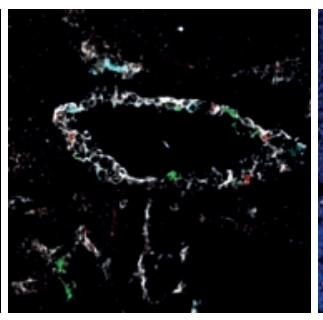

CD31 / DAP

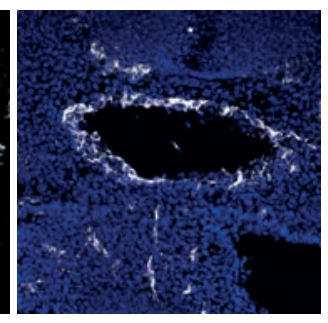

e

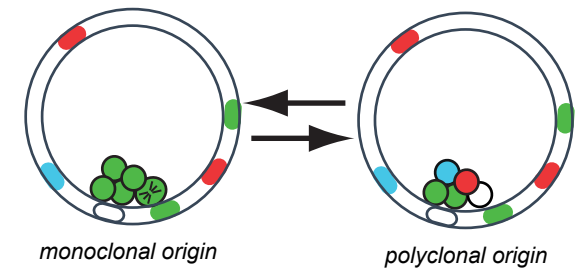

f

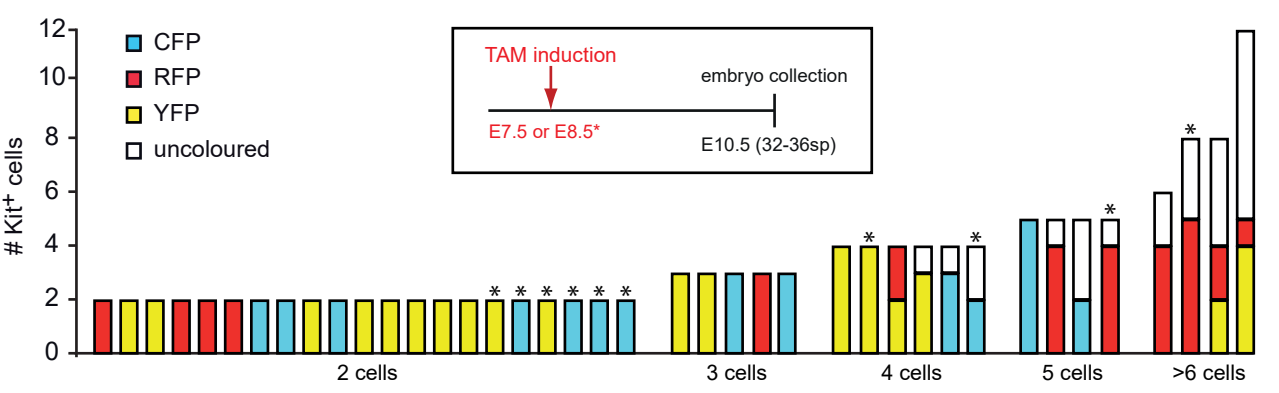

g
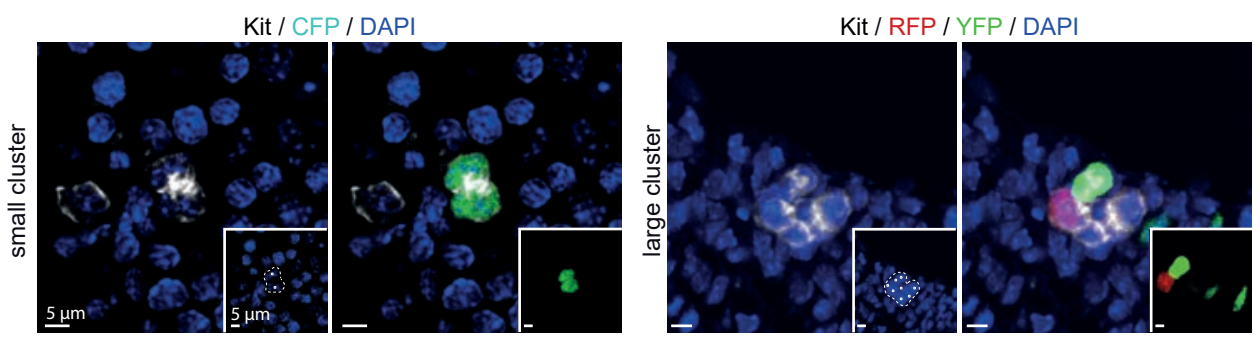
Figure 2: IAHCs have a monoclonal, proliferative origin but become polyclonal at later stage. (a) Schematic representation of time of induction of recombination in VeCadCre ${ }^{\mathrm{ER}}$ :Confetti pregnant females and analysis of embryos. Tamoxifen was injected before cluster initiation (E7.5 or E8.5) and embryos collected at the peak of cluster formation (E10.5 or E11.5). (b) Quantification of recombination efficiency at E10.5 and E11.5 using confocal images. Bars represents the mean \pm SD of the percentage of cells expressing single colored cassettes in CD31+ endothelial cells $(n=2)$. (c,d) Transversal section of a recombined VeCadCre ${ }^{\mathrm{ER}}$ :Confetti AGM from an E10.5 embryo and immunostained with CD31 (c) (white in image, black in label) or Kit (d) (white in image, black in label). Multistack reconstruction of confocal images. Scale bars $30 \mu \mathrm{m}$ (c) and $201 \mathrm{~m}(\mathbf{d})$. (e) Schematic representation of possible outcomes in the IAHC after recombination: Clusters derived from sequential division of one colored cell will be unicolored (left panel). Clusters will be multicolored (right panel) when derived from several, independently recombined endothelial cells. (f) Analyses of recombination in 40 clusters. Bars representing single clusters are grouped based on the number of Kit+ cells. Recombination induction follows the schema in the black square. ( $n=12$ embryo). (g) Representative confetti recombination on clusters of different sizes. Small cluster are monoclonal (left panel), while larger clusters are polyclonal (right panel). Inlet shows relevant confetti channel. Kit is shown in white in the image, in black in the label. Multistack reconstruction of confocal images. Scale bars $5 \mu \mathrm{m}$. 


\section{Figure 3}

a
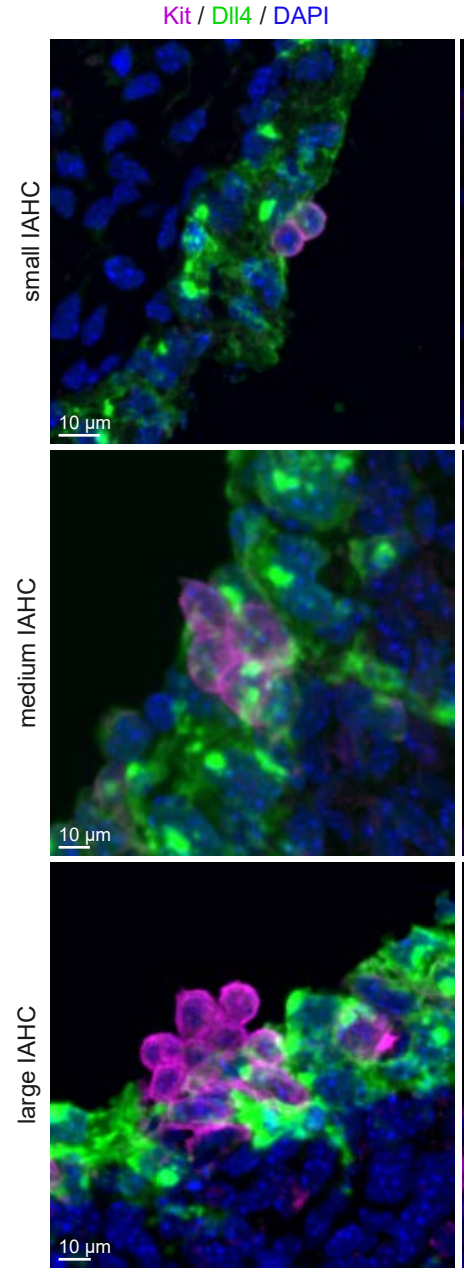

b

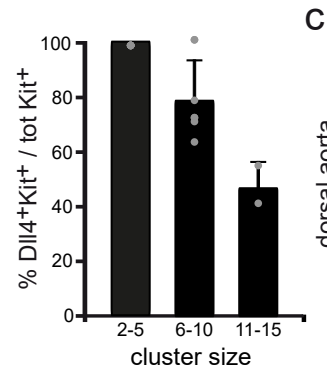

Kit / DAPI
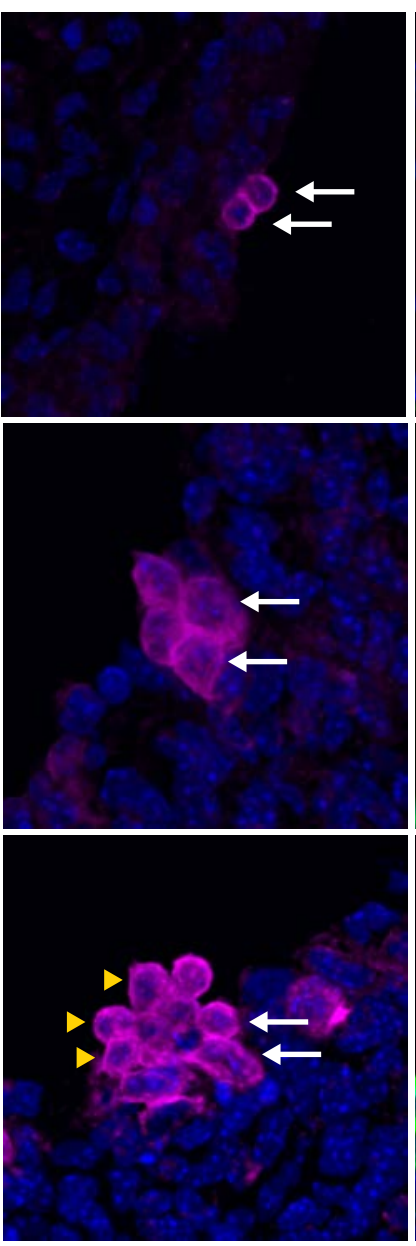

CD31 / DII4 / DAPI

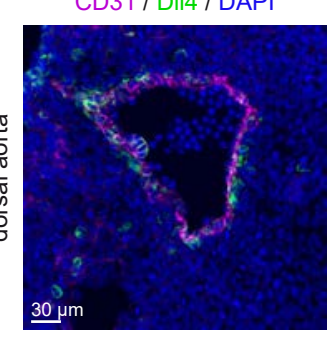

DII4 / DAPI
DII4 / DAPI
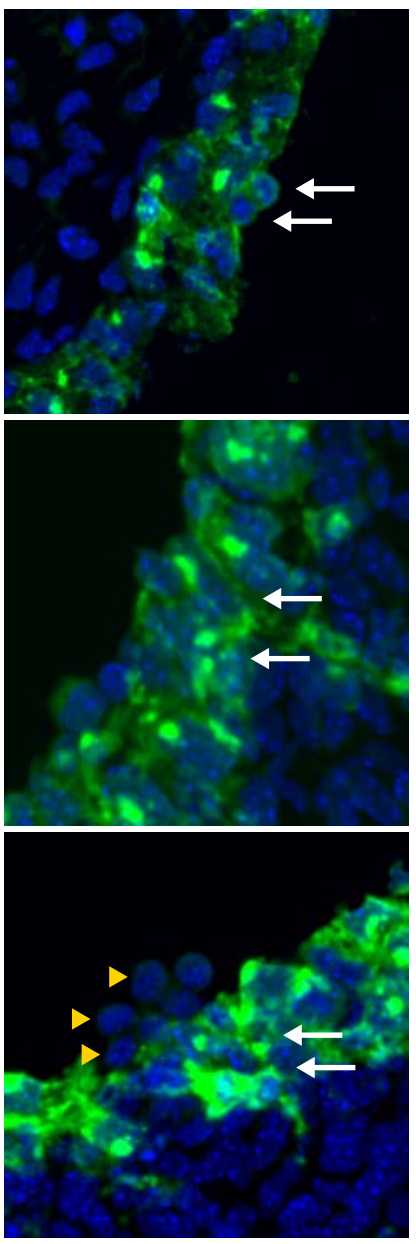

CD31 / DAPI

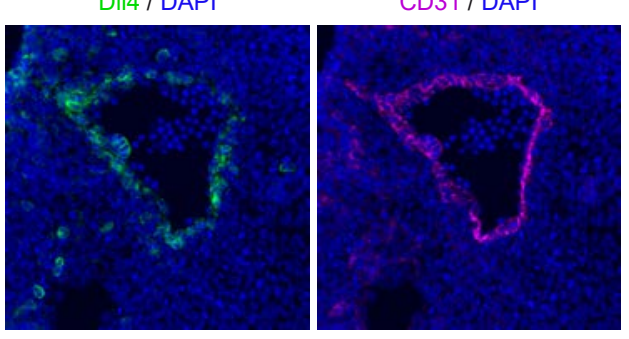


Figure 3: DII4-ligand expression in the IAHC varies with cluster size (a) Detail images of DII4 staining in $\mathrm{Kit}^{+}$cells of IAHC of different sizes. DII4 expression inversely correlates with the size of the cluster: 25 cell IAHCs are uniformly DII4-positive (upper and middle panel), $>5$ cell IAHCs are partially DIl4 positive (lower panel). Multistack reconstruction of confocal images. Scale bars: $10 \mu \mathrm{m}$. (b) Quantification of Kit ${ }^{+}$ DII $4^{+}$cells using confocal images. Bars represent the percentage of DIl4 expressing cells within a cluster in each category of cluster size. Mean $\pm S D$ (total 17 clusters analysed, $n=2$ ). (c) Overview of DII4-ligand expression in the embryonic aorta at E10.5. DII4 is expressed in the majority of the endothelial layer. Scale bar: $30 \mu \mathrm{m}$ 


\section{Figure 4}

a

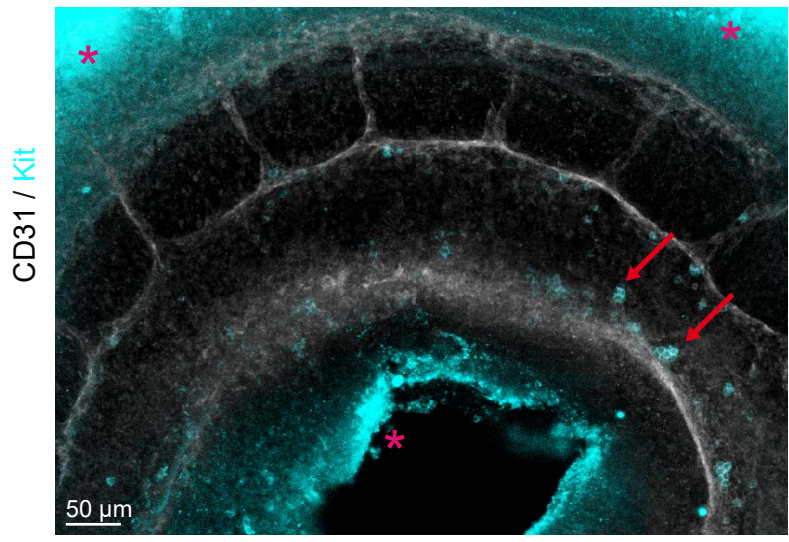

b

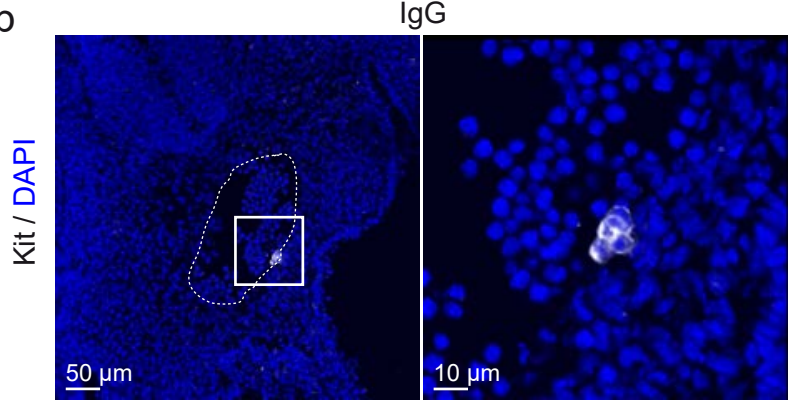

C

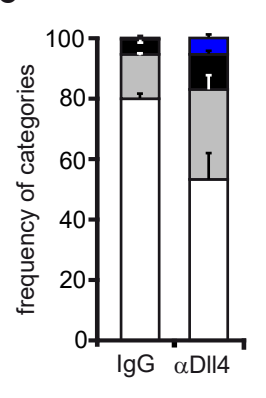

$\square 2-5$ cells d

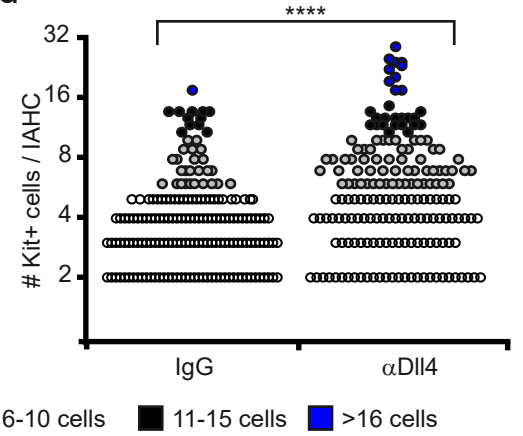

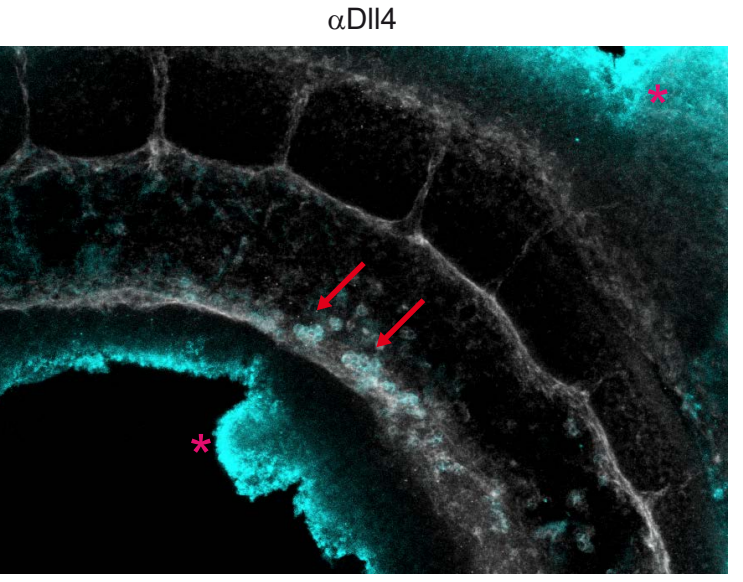

$\alpha \mathrm{D} \| 4$

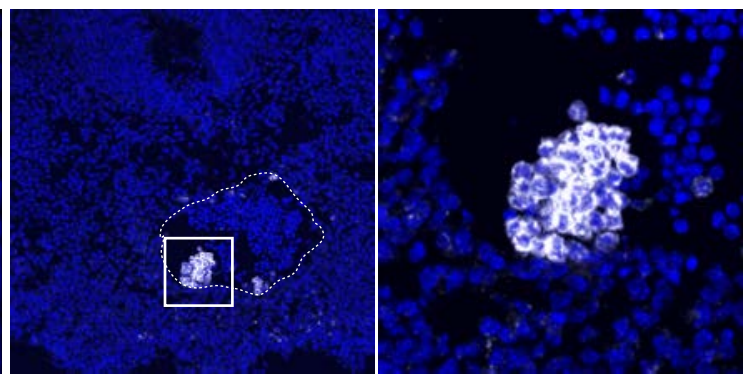

e

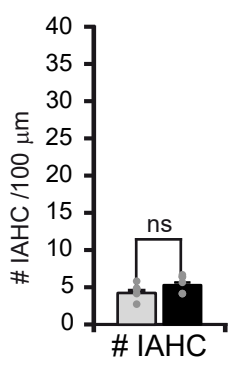

f

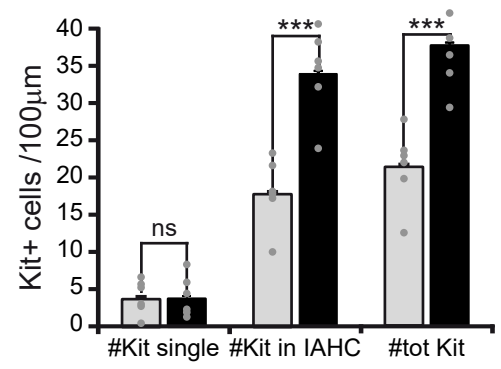

$\square \lg G \square \alpha \mathrm{D} \| 4$ 
Figure 4: Blockage of DII4 increases the number of cells in IAHCs (a)Whole mount images of the aorta endothelium stained for CD31 (white in image, black in label) and Kit (IAHC, cyan) upon IgG (left) or aDII4 antibody treatment (right). Multistack reconstruction of confocal images. Red arrows indicate IAHCs on the ventral part of the aortic wall. Asterisks indicate aspecific autofluorescence. Scale bar: $50 \mu \mathrm{m}$. (b) Representative IAHCs of IgG and $\alpha$ DII4 treated embryos. Kit is shown in white in the image, in black in the label. Multistack reconstruction of confocal images. Note the increased IAHC size in the right panel. Scale bars: $50 \mu \mathrm{m}$ (overview), and $10 \mu \mathrm{m}$ (magnification). (c) Frequency of IAHC categories based on the number of $\mathrm{Kit}^{+}$cells forming a cluster. Quantification of confocal images. Bars represent the mean $\pm S E(n=6)(d)$ Number of Kit+ cells per IAHC increases following $\alpha$ DII4 antibody treatment. Each dot represents one cluster. Quantification on confocal images. Statistical analysis: Mann-Whitney $U$ test. ${ }^{* * * *} p<0.0001$. (e) Total number of IAHCs per $100 \mu \mathrm{m}$. Quantification of confocal images. Mean \pm SE. Statistical analysis: t-test. ns p>0.05; $(n=6)$. (f) Quantification of the number of Kit+ cells per $100 \mu \mathrm{m}$ using confocal images. Bars represent the mean $\pm S E$. Statistical analysis: t-test. $n s p>0.05 ;{ }^{* *} p<0.001(n=6)$. 


\section{Figure 5}

a

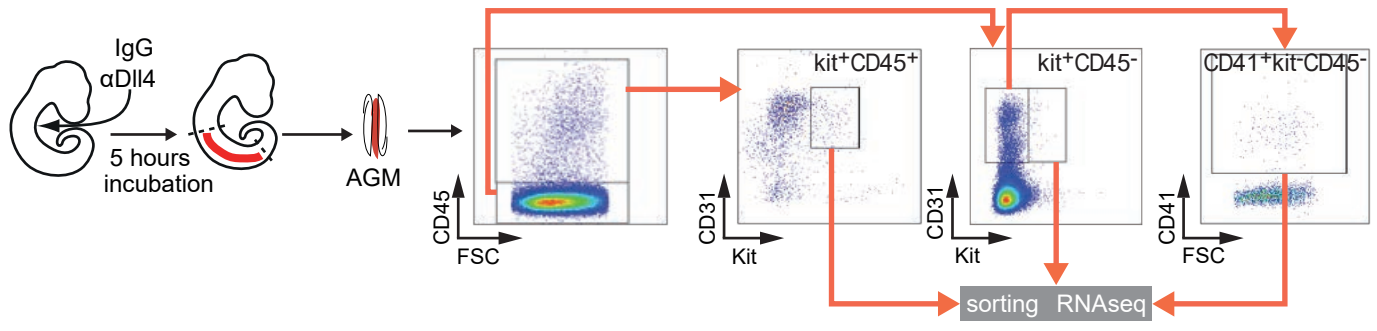

b

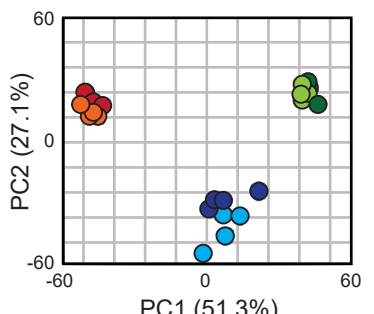

PC1 (51.3\%)

CD41+kit-CD45

$\circ \lg$ O aDII4
C

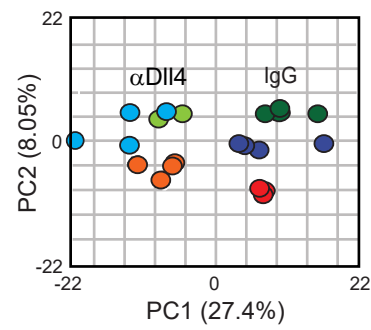

d functional enrichment of DEGs $\alpha D \| 4$ vs IgG ribosome biogenesis
translation
methylation
DNA repair
mRNA processing
RNA splicing
protein transport
TGFbeta receptor

e

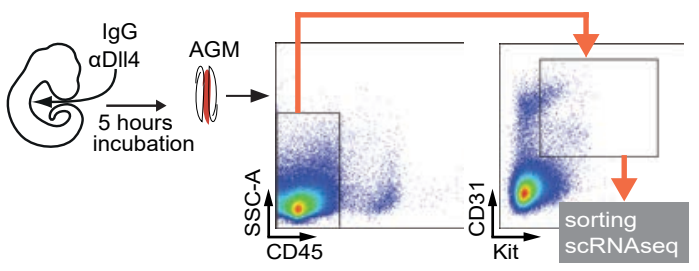

f

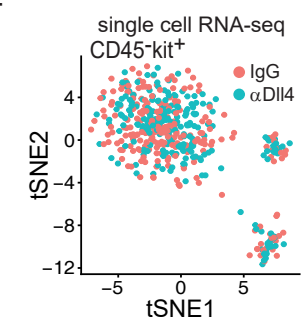

$9 \quad$ ICGS clustering

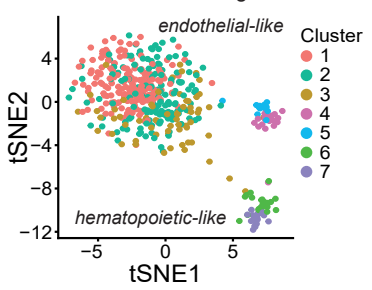

h

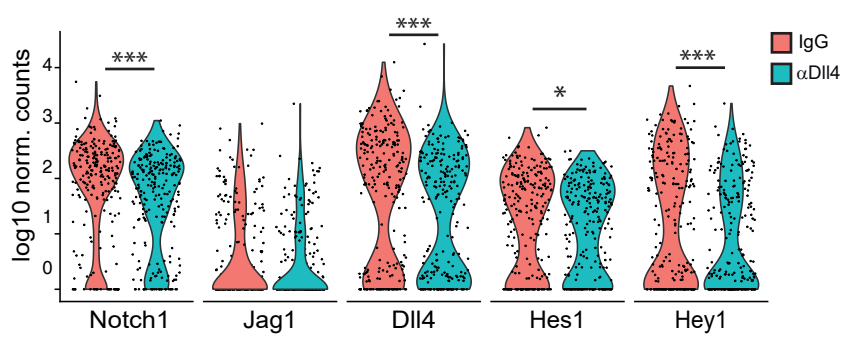

i

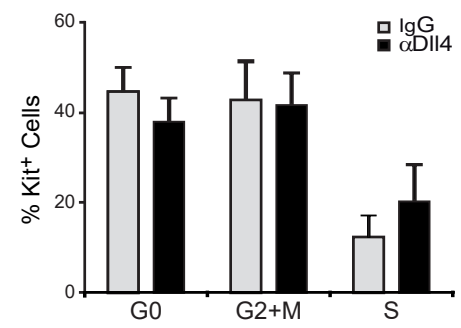


Figure 5: changes in gene expression and decrease in Notch activity after DII4 blockage. (a) Experimental design: E10.5 embryos were injected with IgG or $\alpha$ DII4, incubated for $5 \mathrm{~h}$ and then dissected. The indicated IAHC subpopulations (kit+CD45+; kit+CD45-;kit-CD45-CD41+ were purified and RNA was sequenced. (b) PCA of the different subpopulations from IgG or $\alpha \mathrm{DIl} 4$ treated embryos based on normalized gene counts.(c) PCA considering the DEG according to the treatment specific for each subpopulation. (d) Function enrichment analysis of DEG in the Kit+CD45- population according to the treatment(e) Experimental design and sorting gate for Kit+CD45- cells for single cell RNAseq ( $n=483$ cells).(f-g) tSNE distribution of IgG and $\alpha$ DIl4-treated Kit+CD45- cells from single cell RNA-seq data. (f) cells corresponding to each treatment are indicated and $(\mathbf{g})$ different clusters identified by ICGS (Suppl Fig 5) are represented.1-3 are endothelial-like cells and 6-7 are hematopoietic-like cells.(h) Violin plots of Notch family elements expression in IgG and $\alpha$ DII4 treated cells (adj $p$ val: ${ }^{*}<0.1$ and ${ }^{* * *} p<0.00001$ ). (i) Percentage of cells in each cell cycle phase extracted from single cell RNA-seq analysis. Bars represent the average $\pm S E(n=483)$. 
Figure 6

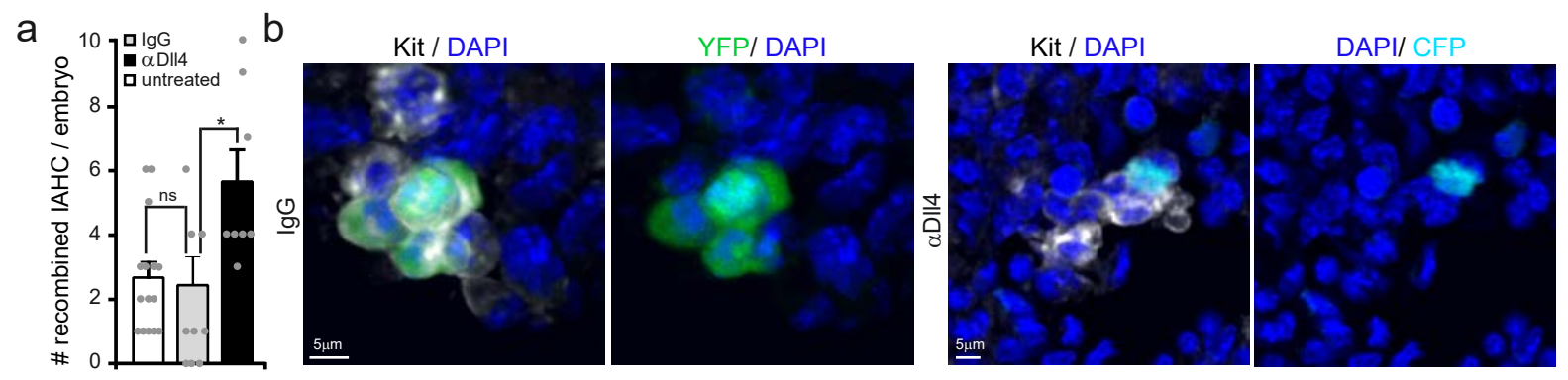

C

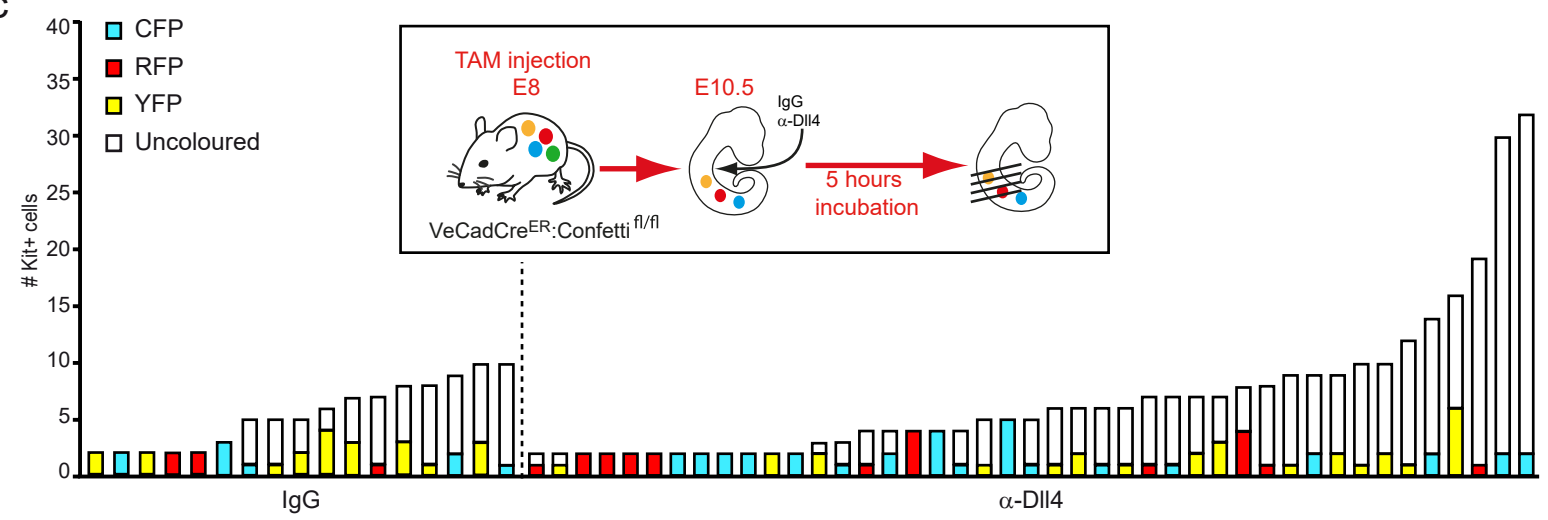

d

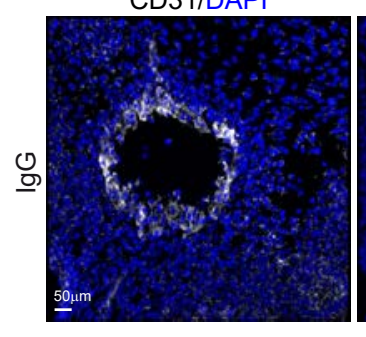

YFP / RFP / CFP / DAPI

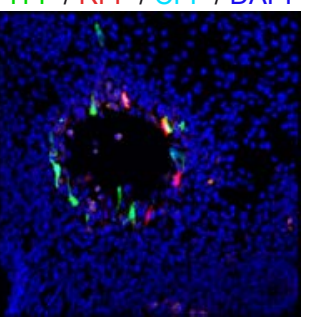

CD31/DAPI

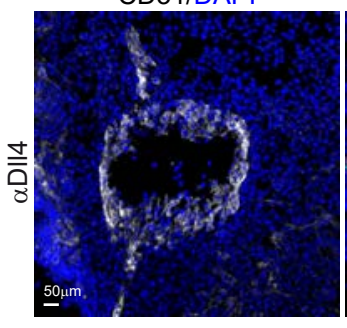

YFP / RFP / CFP / DAPI

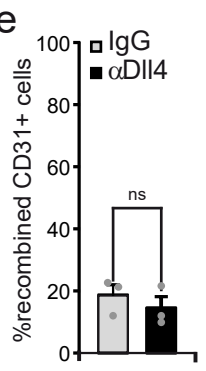




\section{Figure 6: Cell recruitment is increased in DII4-blocked IAHCs}

(a) Bar graph represents the frequency of IAHCs containing recombined cells per embryo under the indicated conditions. Quantification of confocal images. Mean $\pm S E(n=15$ for untreated, $n=7$ for $\lg G$ and $\alpha D \| 4$ treated). (b) Representative images of recombined IAHCs following IgG or $\alpha \mathrm{DIl} 4$ conditions. Kit is shown in white in the image, in black in the label. Multistack reconstruction of confocal images. Scale bar: $5 \mu \mathrm{m}$. (c) Quantification and classification of IAHC containing recombined cells in TAM-induced VeCadCre ${ }^{\mathrm{ER}}$ :Confetti embryos after IgG or $\alpha \mathrm{Dll} 4$ treatment. Embryos treated with $\alpha \mathrm{Dll} 4$ contain more IAHC with colored cells. Quantification of confocal images ( $n=7$ embryos). (d) Representative images of recombined endothelial cells in transversal sections of the aorta. The distribution and amount of recombined endothelial cells is similar in IgG and $\alpha$ DII4 conditions. CD31 is shown in white in the image, in black in the label. Multistack reconstruction of confocal images. Scale bar: 50 $\mu$ m. (e) Quantification of recombined cells in the aortic endothelium using confocal images. Mean $\pm S E(n=3)$. 


\section{Figure 7}

a
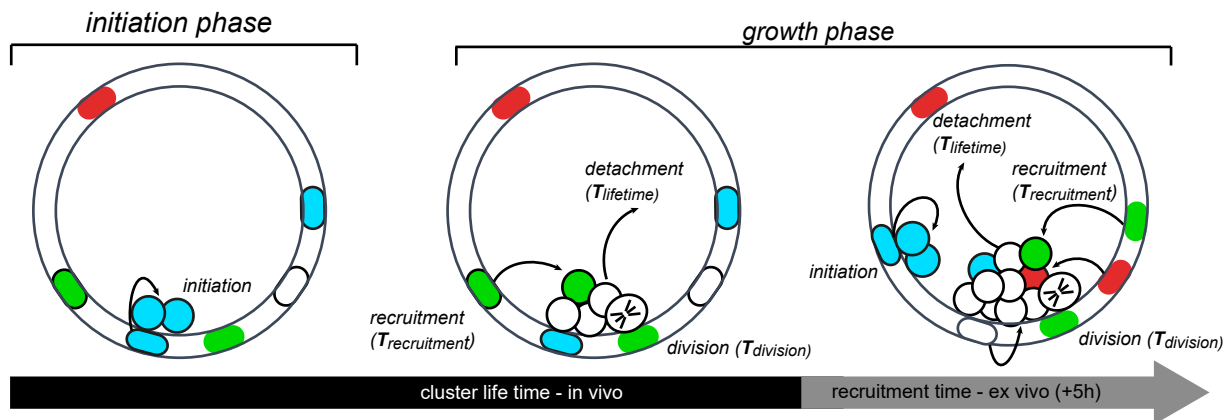

b
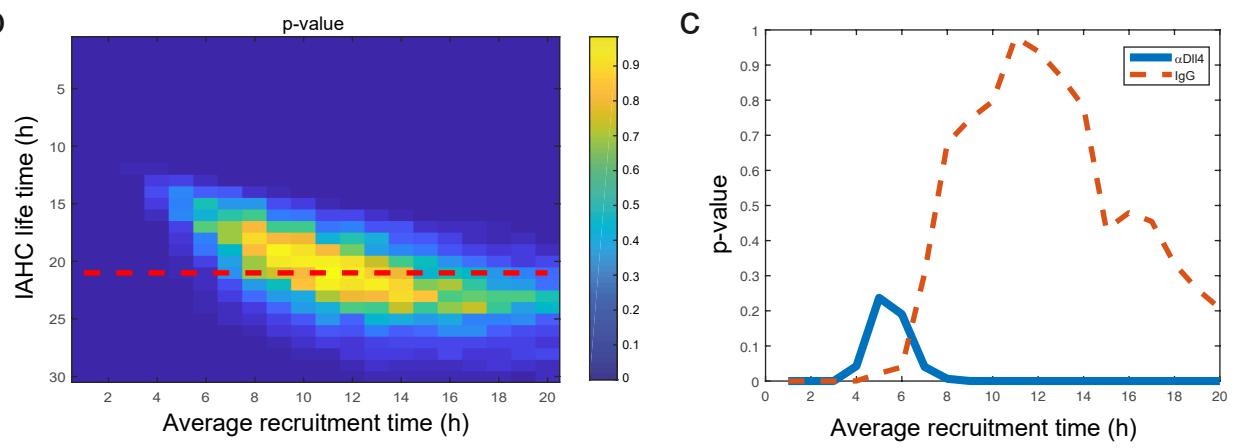

d

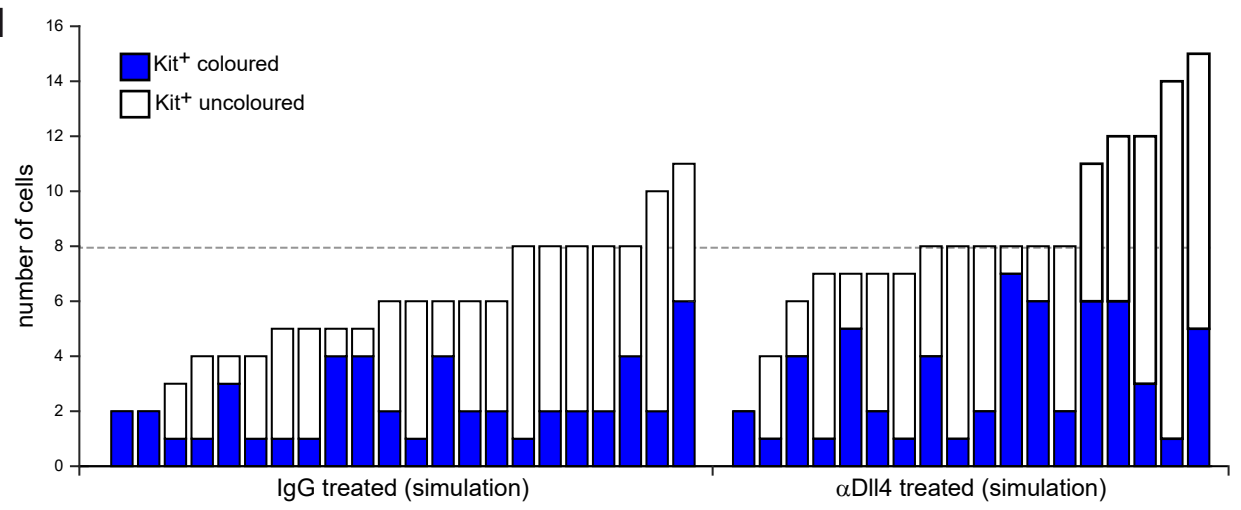

e

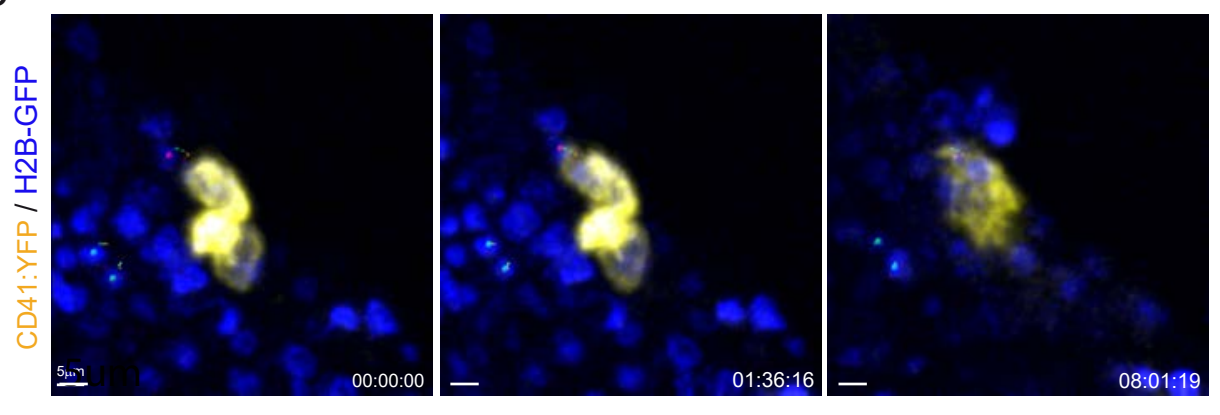


Figure 7: Mathematical model supports a role for DII4 in controlling cell recruitment into IAHCs. (a) Schematics of the probabilistic model of IAHC formation. The processes considered in the model are (1) initiation of cluster, (2) cell division event, (3) recruitment event, and (4) cluster detachment. Each process in the model is described as a typical time scale $\left(T_{\text {cluster lifetime, }}, T_{\text {cell division }}, T_{\text {recruitment }}\right)$. In case of $\alpha D \| 4$ treatment, the final five hours of the simulation are assumed to have a different recruitment time. See details of the simulation in the methods. (b) Heat map showing the probability (or p-value) that the simulation produces mean cluster size and fraction of colored cells that match those observed experimentally for the IgG treatment. This probability is calculated for different values of cluster lifetimes and mean recruitment times (values in $x$ and $y$ ). 4000 simulations were performed for each set of parameters (each point in the heat map). The most likely parameter values (yellow) correspond to a cluster lifetime of 21 hours and mean recruitment time of 11 hours. Red dashed line indicates the crosssection with the most likely cluster lifetime (21 hours). (c) A graph showing the probability that the simulation produces mean cluster size and fraction of colored cells that match those observed experimentally for the aDIl4 treated clusters (blue line). Here, the cluster life time is taken to be 21 hours (taken from b) and the probability to match the experimental data is calculated for different recruitment times in the final 5 hours of the simulation (x-axis). The most likely mean recruitment time for the aDII4 treatment is 5 hours (peak of blue line). For comparison, the most likely mean recruitment time for the lgG treatment is 11 hours (Red dashed line, corresponding to the red dashed in (b)). Hotelling's T-Squared test. (d) Examples of simulations of IAHC distributions for the IgG (left) or aDII4 (right) treated samples. Simulations were performed with the most likely parameter values described in $b$ and $c$. Note that only clusters that have at least one colored cell are shown (i.e clusters with non-colored cells are not represented).(e) Snapshots of Movie 5. Time Lapse of embryonic organotypic slice from a CD41:YFP: H2B-GFP reporter mouse showing the recruitment of CD41:YFP negative cells (magenta central pointtracking analyses) migrating towards the IAHC (CD41:YFP+ cells). Green central point and relative tracking identify cells with non-directional movement. Scale bar: $5 \mu \mathrm{m}$. Time expressed in hh:mm:ss. 


\section{Figure 8}

a

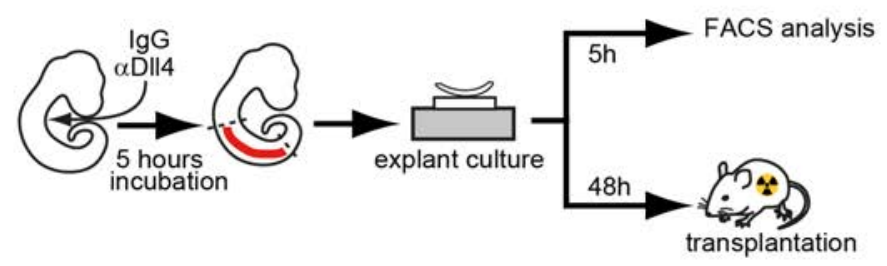

b

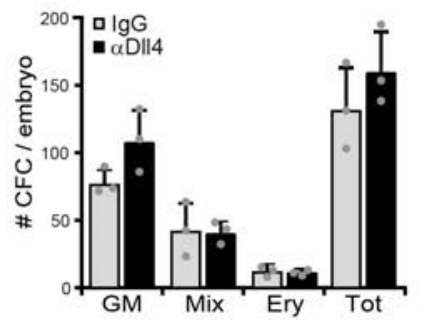

d

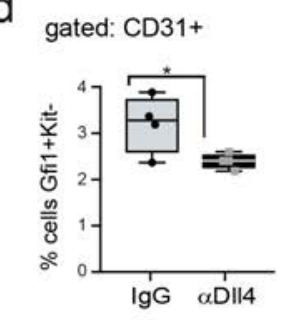

e

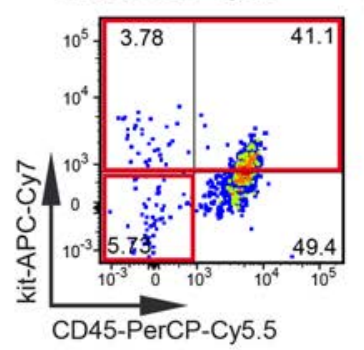

gated: $\mathrm{CD} 31+$

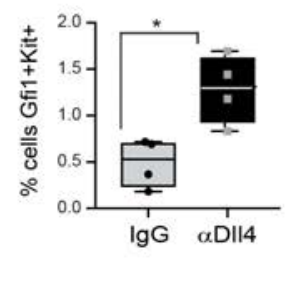

f

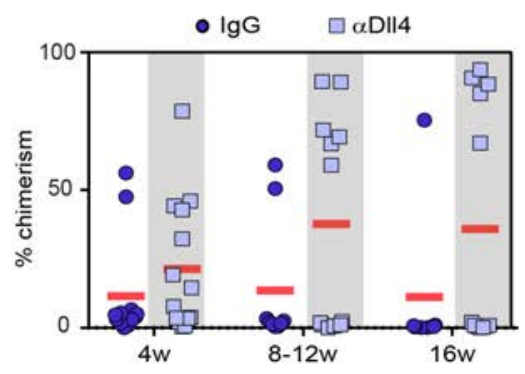

g

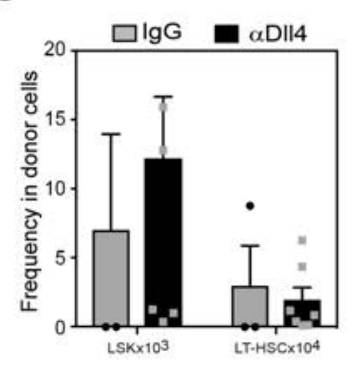

$\mathrm{h}$

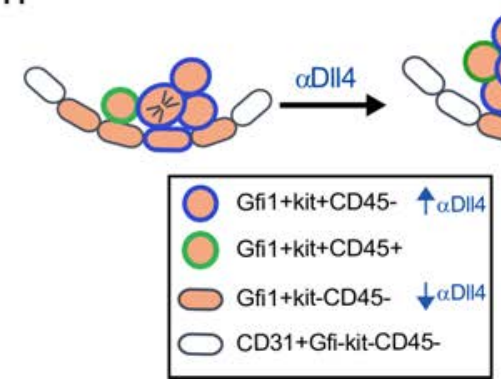

Figure 8: Interference with the DIl4 ligand functionality results in increased hematopoietic potential in embryonic AGM. (a) Schematic representation of functional assays for testing the hematopoietic potential of AGM. Embryos were injected intracardiacally and incubated for five hours with $\alpha \mathrm{DII} 4$ or IgG control. Explant cultures were either exposed to the relevant treatment for $5 \mathrm{~h}$ before FACS analyses or incubated for $48 \mathrm{~h}$ before transplantation into irradiated mice. (b) Colony-forming assays using E10.5 AGMs derived from embryos treated with IgG or $\alpha$ DIl4. Mean $\pm S D(n=3)$ No significant differences were detected (c-e) FACS analyses of Gfi1 ${ }^{+}$cells derived from explants treated with IgG or $\alpha$ Dll4 for 5 hours (c). Analyses of the hemogenic Gfi1 ${ }^{+} \mathrm{Kit}(\mathbf{d})$ and the $\mathrm{Gfi}^{+} \mathrm{Kit}^{+}(\mathbf{e})$ within the CD31+ ${ }^{+} \mathrm{CD} 45^{-}$ population. Statistical analysis: t-test. Box plots show upper and lower quartile; center line represents mean $(n=4)$. ${ }^{*} p<0.05 ;{ }^{* *} p<0.005$; non significant comparisons are not indicated (f) Percentage of 
reconstitution (chimerism determined by CD45.1 and CD45.2 expression) in peripheral blood (PB). Embryos treated with IgG or $\alpha \mathrm{DIl} 4$ were kept in explant culture for 48hours and then transplanted into irradiated recipients. Peripheral blood was analyzed at different time points. Each dot/square represents a single transplanted mouse. (g) Analysis of progenitors (lin-sca1+kit+) and HSCs (Lin-sca1+kit+CD48CD150+) in the donor population in the bone marrow after 4 months of transplantation. (h) Model for recruitment of $\mathrm{Gfi}^{+}$cells into the IAHC. Recruitment of $\mathrm{Gfi}^{+}$endothelial cells increases upon DII4 blockage. 